\title{
S2k-Leitlinie Qualitätsanforderungen in der gastrointestinalen Endoskopie (Kurzversion), AWMF Register Nr. 021-022 Erstauflage 2015
}

\section{S2k guideline: quality requirements for gastrointestinal endoscopy, AWMF registry no. 021-022}

Autoren

Institute
U. Denzer ${ }^{1}$, U. Beilenhoff ${ }^{2}$, A. Eickhoff ${ }^{3}$, S. Faiss ${ }^{4}$, P. Hüttl ${ }^{5}$, S. In der Smitten ${ }^{6}$, R. Jakobs ${ }^{7}$, C. Jenssen ${ }^{8}$, M. Keuchel ${ }^{9}$ F. Langer ${ }^{10}$, M. M. Lerch ${ }^{11}$, P. Lynen Jansen ${ }^{12}$, A. May ${ }^{13}$, R. Menningen ${ }^{14}$, G. Moog ${ }^{15}$, T. Rösch ${ }^{16}$, U. Rosien ${ }^{17}$, T. Vowinkel ${ }^{14}$, T. Wehrmann ${ }^{18}$, U. Weickert ${ }^{18}$

Die Institutsangaben sind am Ende des Beitrags gelistet.

\section{Bibliografie}

DOI http://dx.doi.org/ 10.1055/s-0041-109520

Z Gastroenterol 2015; 53: 1496-1530 @ Georg Thieme Verlag KG Stuttgart · New York . ISSN 0044-2771

\section{Korrespondenzadresse Priv.-Doz. Dr. med Ulrike Denzer}

Klinik und Poliklinik für Interdisziplinäre Endoskopie, Universitätsklinikum HamburgEppendorf

Martinistr. 52

20246 Hamburg

Tel.: ++49/40/741050098

Fax: ++ 49/40/741040004

u.denzer@uke.de

\begin{tabular}{|c|c|c|}
\hline \multicolumn{2}{|c|}{ Inhaltsverzeichnis } & \multirow{2}{*}{$\begin{array}{l}\text { Seite } \\
1497\end{array}$} \\
\hline Kap. 1 & $\begin{array}{l}\text { Informationen zur Kurzversion und } \\
\text { methodische Hinweise }\end{array}$ & \\
\hline Kap. 2 & Strukturqualität & 1498 \\
\hline Kap. 2.1 & Endoskope/Instrumente & 1498 \\
\hline 2.1 .1 & Endoskope & 1498 \\
\hline 2.1 .2 & Instrumente & 1498 \\
\hline Kap. 2.2 & $\begin{array}{l}\text { Räumlich-apparative } \\
\text { Voraussetzungen }\end{array}$ & 1498 \\
\hline 2.2 .1 & $\begin{array}{l}\text { Endoskopieeinheit: Raumbedarf, } \\
\text { Raumnutzung und Größe }\end{array}$ & 1498 \\
\hline 2.2 .2 & $\begin{array}{l}\text { Endoskopieeinheit: Raumausstattung } \\
\text { der Eingriffsräume }\end{array}$ & 1498 \\
\hline 2.2 .3 & $\begin{array}{l}\text { Endoskopieeinheit: Raumausstattung } \\
\text { Aufbereitungsraum }\end{array}$ & 1499 \\
\hline 2.2 .4 & $\begin{array}{l}\text { Endoskopieeinheit: Raumausstattung } \\
\text { von Aufwachbereich, Wartebereich } \\
\text { und Empfangsbereich }\end{array}$ & 1499 \\
\hline 2.2 .5 & Endoskopieeinheit: Weitere Räume & 1499 \\
\hline Kap. 2.3 & $\begin{array}{l}\text { Personelle Ausstattung in der } \\
\text { gastroenterologischen Endoskopie }\end{array}$ & 1499 \\
\hline 2.3 .1 & Prozessmessung & 1499 \\
\hline 2.3 .2 & Arbeitsplatzmethode & 1499 \\
\hline Kap. 2.4 & $\begin{array}{l}\text { Definition eines Standards für } \\
\text { endoskopische Prozeduren für } \\
\text { Endoskopiefachberufe }\end{array}$ & 1499 \\
\hline 2.4 .1 & $\begin{array}{l}\text { Pflegestandards als Instrumente des } \\
\text { Qualitätsmanagements (QM) }\end{array}$ & 1499 \\
\hline 2.4 .2 & $\begin{array}{l}\text { Strukturqualität für endoskopie- } \\
\text { spezifische Standards }\end{array}$ & 1499 \\
\hline 2.4 .3 & $\begin{array}{l}\text { Prozessqualität für endoskopie- } \\
\text { spezifische Standards }\end{array}$ & 1500 \\
\hline 2.4 .4 & $\begin{array}{l}\text { Ergebnisqualität für endoskopie- } \\
\text { spezifische Standards }\end{array}$ & 1500 \\
\hline 2.4 .5 & Zusammenfassung & 1501 \\
\hline Kap. 3 & $\begin{array}{l}\text { Prozessqualität - Patientenvorbe- } \\
\text { reitung }\end{array}$ & 1501 \\
\hline Kap 3.1 & Aufklärung endoskopischer Eingriffe & 1501 \\
\hline 3.1 .1 & $\begin{array}{l}\text { Aushändigung der Patienten- } \\
\text { aufklärung an den Patienten }\end{array}$ & 1501 \\
\hline 3.1 .2 & Arten der Aufklärung & 1501 \\
\hline 3.1 .3 & Das Aufklärungsgespräch & 1501 \\
\hline 3.1.4 & Zeitpunkt der Aufklärung & 1501 \\
\hline 3.1 .5 & Inhalt des Aufklärungsgespräches & 1501 \\
\hline 3.1 .6 & Aufklärungsadressat & 1501 \\
\hline
\end{tabular}




\begin{tabular}{|c|c|c|}
\hline \multicolumn{2}{|c|}{ Inhaltsverzeichnis } & \multirow{2}{*}{$\begin{array}{l}\text { Seite } \\
1508\end{array}$} \\
\hline Kap. 4.3 & Endoskopische Varizenbehandlung & \\
\hline 4.3 .1 & Endoskopisches Varizenscreening & 1508 \\
\hline 4.3 .2 & Elektive endoskopische Varizenbehandlung & 1508 \\
\hline 4.3.3 & Akute Varizenblutung & 1509 \\
\hline 4.3.4 & Komplikationen & 1510 \\
\hline 4.3 .5 & Spezifische Qualitätsindikatoren & 1510 \\
\hline Kap. 4.4 & Endoskopische Therapie nicht variköser Blutungen & 1510 \\
\hline 4.4 .1 & Zeitpunkt der Endoskopie & 1510 \\
\hline 4.4 .2 & Spezielle Vorbereitung & 1510 \\
\hline 4.4 .3 & Durchführung & 1510 \\
\hline 4.4.4 & Prozedurabhängige Nachsorge & 1511 \\
\hline 4.4 .6 & Spezifische Qualitätsindikatoren & 1511 \\
\hline Kap. 4.5 & Koloskopie & 1511 \\
\hline 4.5 .1 & Spezielle Kontraindikationen & 1511 \\
\hline 4.5 .2 & Spezielle Vorbereitung/Voraussetzungen & 1511 \\
\hline 4.5 .3 & Durchführung & 1512 \\
\hline 4.5.4 & Spezifische Qualitätsindikatoren & 1513 \\
\hline Kap. 4.6 & Endoskopische Resektion & 1513 \\
\hline 4.6.1 & Spezielle Vorbereitung & 1513 \\
\hline 4.6 .2 & Durchführung & 1514 \\
\hline 4.6 .3 & Prozedurabhängige Nachsorge & 1514 \\
\hline Kap. 4.7 & $\begin{array}{l}\text { Endoskopische enterale Dilatation, Bougierung } \\
\text { und Stenteinlage in der Therapie von Stenosen } \\
\text { in Ösophagus, Magen, Duodenum, Kolon und } \\
\text { postoperativen Leckagen }\end{array}$ & 1514 \\
\hline 4.7 .1 & Spezielle Vorbereitung & 1514 \\
\hline 4.7 .2 & Durchführung & 1515 \\
\hline 4.7 .3 & Prozedurabhängige Nachsorge & 1516 \\
\hline Kap. 4.8 & ERCP & 1516 \\
\hline 4.8 .1 & Spezielle Kontraindikationen & 1516 \\
\hline 4.8 .2 & Spezielle Vorbereitung & 1516 \\
\hline 4.8 .3 & Durchführung & 1517 \\
\hline 4.8 .4 & Prozedurabhängige Nachsorge & 1519 \\
\hline 4.8 .5 & Spezifische Qualitätsindikatoren ERCP & 1519 \\
\hline Kap. 4.9 & $\begin{array}{l}\text { Diagnostische Endosonografie und } \\
\text { endosonografisch gestützte Feinnadelpunktion }\end{array}$ & 1519 \\
\hline 4.9.1 & Spezielle Kontraindikationen & 1519 \\
\hline 4.9 .2 & Spezielle Vorbereitung & 1520 \\
\hline 4.9 .3 & Durchführung & 1520 \\
\hline 4.9 .4 & Prozedurabhängige Nachsorge & 1520 \\
\hline 4.9 .5 & Spezifische Qualitätsindikatoren EUS & 1521 \\
\hline Kap. 4.10 & $\begin{array}{l}\text { EUS-gestützte Drainage pankreatitisassoziierter } \\
\text { Flüssigkeitsansammlungen }\end{array}$ & 1521 \\
\hline 4.10 .1 & Spezielle Vorbereitung & 1521 \\
\hline 4.10 .2 & Durchführung & 1521 \\
\hline Kap. 4.11 & $\begin{array}{l}\text { Addendum EUS-gestützte therapeutische } \\
\text { Interventionen }\end{array}$ & 1522 \\
\hline 4.11 .1 & $\begin{array}{l}\text { EUS-gestützte prätherapeutische } \\
\text { Tumormarkierung }\end{array}$ & 1522 \\
\hline 4.11 .2 & $\begin{array}{l}\text { EUS-gestützte Blockade/Neurolyse des Plexus } \\
\text { coeliacus }\end{array}$ & 1522 \\
\hline 4.11 .3 & $\begin{array}{l}\text { EUS-gestützte Gallengangs- und } \\
\text { Pankreasgangdrainage }\end{array}$ & 1522 \\
\hline 4.11 .4 & EUS-gestützte Tumortherapie & 1522 \\
\hline Kap. 4.12 & PTCD & 1523 \\
\hline 4.12 .1 & Spezielle Kontraindikationen & 1523 \\
\hline 4.12 .2 & Spezielle Vorbereitung & 1523 \\
\hline 4.12 .3 & Durchführung & 1523 \\
\hline 4.12 .4 & Prozedurabhängige Nachsorge & 1523 \\
\hline 4.12 .5 & Spezifische Qualitätsindikatoren PTCD & 1524 \\
\hline Kap. 4.13 & Flexible Dünndarmenteroskopie & 1524 \\
\hline 4.13 .1 & Spezielle Kontraindikationen & 1524 \\
\hline 4.13 .2 & Spezielle Vorbereitung & 1524 \\
\hline 4.13 .3 & Durchführung & 1524 \\
\hline 4.13.4 & Prozedurabhängige Nachsorge & 1524 \\
\hline
\end{tabular}

\begin{tabular}{|lll|}
\hline Inhaltsverzeichnis & Seite \\
\hline Kap.4.14 & Kapselendoskopie & 1524 \\
\hline 4.14 .1 & Spezielle Kontraindikationen & 1524 \\
\hline 4.14 .2 & Spezielle Vorbereitung & 1524 \\
\hline 4.14 .3 & Durchführung & 1525 \\
\hline 4.14 .4 & Prozedurabhängige Nachsorge & 1525 \\
\hline 4.14 .5 & Spezifische Qualitätsindikatoren Kapselendoskopie & 1525 \\
\hline Kap.4.15 & Diagnostische Laparoskopie & 1525 \\
\hline 4.15 .1 & Spezielle Kontraindikationen & 1525 \\
\hline 4.15 .2 & Spezielle Vorbereitung & 1525 \\
\hline 4.15 .3 & Durchführung & 1525 \\
\hline 4.15 .4 & Prozedurabhängige Nachsorge & 1526 \\
\hline 4.15 .5 & Komplikationen der diagnostischen Laparoskopie & 1526 \\
\hline Kap.4.16 & Endoskopie in der Schwangerschaft & 1526 \\
\hline Kap.5 & Prozessqualität - Dokumentation von Befunden & 1526 \\
\hline 5.1 & Befunddokumentation & 1526 \\
\hline 5.2 & Bild-/Filmdokumentation & 1526 \\
\hline Kap.6 & Prozessqualität - Hygiene und Aufbereitung & 1526 \\
\hline 6.1 & Strukturqualität für Hygiene in der Endoskopie & 1527 \\
\hline 6.1 .1 & Sicherheit der Patienten & 1527 \\
\hline 6.1 .2 & Sicherheit des Personals & 1527 \\
\hline 6.1 .3 & Ausbildung und Kompetenzen von Personal & 1527 \\
\hline 6.1 .4 & Anzahl des Personals & 1527 \\
\hline 6.1 .5 & Räumlich-apparative Voraussetzungen & 1527 \\
\hline 6.2 & Prozessqualität zur Hygiene in der Endoskopie & 1527 \\
\hline 6.2 .1 & Hygienemaßnahmen während & 1527 \\
\hline 6.2 .2 & Untersuchungen und Eingriffen & \\
\hline 6.3 & Hygienemaßnahmen während der Aufbereitung & 1527 \\
\hline 6.4 & von Medizinprodukten in der Endoskopie & 1528 \\
\hline Kap.7 & Ergebnisqualität der Hygiene in der Endoskopie & 1528 \\
\hline & Ergebnisqualität und Allgemeine & 1529 \\
\hline & Qualitätsindikatoren Endoskopie & \\
\hline & & \\
\hline & & \\
\hline
\end{tabular}

\section{Kap. 1 Informationen zur Kurzversion}

Bei diesem Dokument handelt es sich um die Kurzversion der S2kLeitlinie Qualitätsanforderungen in der Gastrointestinalen Endoskopie, welche über die folgenden Seiten zugänglich ist:

- DGVS (www.dgvs.de)

- AWMF (www.leitlinien.net)

Die Langversion der Leitlinie, die auch den Leitlinienreport enthält, ist ebenfalls über diese Seiten verfügbar oder kann über www.thieme-connect.de/products/ejournals/journal/10.1055/s00000094 abgerufen werden.

Die Leitlinie wurde unter Federführung der Deutschen Gesellschaft für Gastroenterologie (DGVS) unter Beteiligung der Deutsche Morbus Crohn/Colitis ulcerosa Vereinigung e. V (DCCV), der Deutsche Gesellschaft für Endoskopie und Bildgebende Verfahren (DGE-BV), der Deutsche Gesellschaft für Allgemein- und Viszeralchirurgie (DGAV), der Chirurgische Arbeitsgemeinschaft für Endoskopie und Sonografie (CAES), des Berufsverband niedergelassener Gastroenterologen Deutschlands e.V. (bng), der Deutsche Gesellschaft für Thrombose und Hämostaseforschung und der Deutsche Gesellschaft für Endoskopie - Assistenzpersonal e.V. (DEGEA) erstellt.

P. Baltes, Hamburg, K. Fraedrich, Hamburg, A. Gaus, Hamburg, B. Hartmann, Ludwigshafen und M. Jung, Mainz wurden als weitere Experten bei der Erstellung der Beiträge eingebunden.

Für das Kapitel Aufklärung erfolgte eine juristische Beratung durch Dr. P. Hüttl, Rechtsanwaltskanzlei Dr. Heberer \& Kollegen München. 
Alle Teilnehmer legten ihre potenziellen Interessenskonflikte offen. Der Umgang mit potenziellen Interessenskonflikten ist in der Langversion der Leitlinie dargelegt.

Alle Empfehlungen wurden auf Basis einer Evidenzrecherche in einem zweistufigen Konsensusverfahren von der Leitliniengruppe verabschiedet ( $\bullet$ Tab. 1 ).

Tab. 1 Schema zur Graduierung von Empfehlungen.

\begin{tabular}{|ll}
\hline Syntax & Beschreibung \\
\hline soll & starke Empfehlung \\
\hline sollte & Empfehlung \\
\hline kann & Empfehlung offen \\
\hline
\end{tabular}

Die Leitlinie wurde allen beteiligten Fachgesellschaften und der Patientenvertretung zur Stellungnahme vorgelegt und von diesen verabschiedet. Durch die AWMF erfolgte eine externe formale Beurteilung.

Die Gültigkeit der Leitlinie beträgt 5 Jahre (Juli 2020). Eine Überarbeitung der Leitlinie bei veränderter Datenlage erfolgt gegebenenfalls auch früher.

\section{Kap. 2 Strukturqualität}

$\nabla$

Kap. 2.1 Endoskope/Instrumente

2.1.1 Endoskope

Statement:

Die Grundtechnik der im Gastrointestinaltrakt verwendeten Endoskope ist zwischen den verschiedenen Anbietern vergleichbar. Starker Konsens

\section{Empfehlung:}

Die verwendeten Endoskope sollen Videotechnik aufweisen, Fiberglasendoskope sollten wegen der deutlich schlechteren Bildqualität außerhalb von Spezialanwendungen (z. B. Cholangioskopie) nicht mehr verwendet werden.

Starker Konsens

\subsubsection{Instrumente}

Empfehlung:

Einmalprodukte sollen nicht mehrfach verwendet werden.

Starker Konsens

\subsection{Räumlich-apparative Voraussetzungen}

\subsubsection{Endoskopie Einheit: Raumbedarf, Raumnutzung} und Größe

Empfehlung:

Endoskopische Eingriffe sollen in dafür eingerichteten Räumlichkeiten durchgeführt werden, die alle Geräte und Instrumente für den Eingriff selbst, die Patientenbetreuung, Sedierung, Überwachung, Notfallmanagement bereithalten.

Bei gegebener Indikation können endoskopische Untersuchungen außerhalb der eigentlichen Endoskopieeinheit stattfinden, insbesondere auf der Intensivstation oder auch im Operationssaal.

Starker Konsens

Empfehlung:

Die Größe der Endoskopieeinheit und die Anzahl der Eingriffsräume soll sich an der Anzahl und Art der durchzuführenden Untersuchungen orientieren.

Starker Konsens

\section{Empfehlung:}

In klinischen Einrichtungen mit entsprechendem Untersuchungsaufkommen soll mindestens ein Raum mit der Möglichkeit der Durchleuchtung vorhanden sein.

Starker Konsens

\section{Empfehlung:}

In klinischen Einrichtungen mit entsprechendem Untersuchungsaufkommen soll mindestens ein Raum die Voraussetzungen für die Durchführung endoskopischer Interventionen in Allgemeinanästhesie aufweisen

Starker Konsens

\section{Empfehlung:}

Die endoskopischen Eingriffsräume sollen eine ausreichende Größe besitzen. Insbesondere bei Neuplanung einer Endoskopieeinheit wird eine Mindestgröße von $30 \mathrm{~m}^{2}$ für einen Eingriffsraum und $36 \mathrm{~m}^{2}$ für einen Eingriffsraum mit Durchleuchtung empfohlen.

Starker Konsens

\subsubsection{Endoskopieeinheit: Raumausstattung der Eingriffsräume \\ Empfehlung:}

In den endoskopischen Eingriffsräumen, im Aufwachraum, im Wartebereich und in der Aufbereitung soll der Fußbodenbelag flüssigkeitsdicht verfugt, abwaschbar und desinfizierbar sein. In den endoskopischen Eingriffsräumen soll der Wandbelag abwaschbar und desinfizierbar bis mindestens $2 \mathrm{~m}$ Höhe sein.

Starker Konsens

\section{Empfehlung:}

In den Eingriffsräumen sollte durch geeignete Lüftungs- bzw. Klimaanlagen eine ausreichende Frischluftzufuhr und Temperaturregelung ermöglicht werden.

Starker Konsens

\section{Empfehlung:}

In den Eingriffsräumen sollen eine Abdunkelungsvorrichtung sowie eine ausreichend helle stufenweise abregelbare Lichtquelle vorhanden sein.

Starker Konsens

\section{Empfehlung:}

Bei Neuplanung sollten die Eingriffsräume mit Wandanschlüssen für alle medizinisch notwendigen Versorgungsleitungen $\left(\mathrm{O}_{2} ; \mathrm{Co}_{2}\right.$, Vakuumabsaugung, Steckdosen) ausgestattet sein.

Starker Konsens

\section{Empfehlung:}

Jeder endoskopische Eingriffsraum soll über einen hygienischen Händewaschplatz verfügen.

Starker Konsens

\section{Empfehlung:}

In den Eingriffsräumen sollten Kabel in Kabelkanälen verlegt werden.

Starker Konsens

\section{Empfehlung:}

Jeder endoskopische Eingriffsraum soll mit einem hygienischen Händewaschplatz ausgestattet sein. Ein Umkleideraum für den 
Patienten sollte angeschlossen sein. Eine Patiententoilette mit Waschbecken und Händedesinfektion kann angeschlossen sein. Starker Konsens

\section{Empfehlung:}

Jeder endoskopische Eingriffsraum sollte mit einem Befundungsplatz mit entsprechender zentral vernetzter EDV ausgestattet sein. Alternativ kann ein zentraler Befundungsraum eingerichtet sein.

Starker Konsens

\section{Empfehlung:}

Jeder endoskopische Eingriffsraum soll mit einer Monitoreinheit zur kardiopulmonalen Überwachung von sedierten Patienten ausgestattet sein. Sämtliche technische Hilfsmittel und Medikamente zur Beherrschung kardiorespiratorischer Komplikationen sollen vor Ort zur Verfügung stehen.

Starker Konsens

\section{Empfehlung:}

Die Anordnung des Endoskopieequipments, des Patienten und des Untersuchers soll ein ergonomisches Arbeiten des Untersuchers und des Assistenzpersonals ermöglichen

Starker Konsens

\section{Empfehlung:}

Die Ausstattung der Eingriffsräume soll an die durchzuführenden Untersuchungen angepasst sein.

Starker Konsens

\section{Empfehlung:}

Die Ausstattung des Durchleuchtungsraumes soll den Anforderungen des Strahlenschutzes entsprechen.

Starker Konsens

\subsubsection{Endoskopieeinheit: Raumausstattung \\ Aufbereitungsraum \\ Empfehlung:}

Die räumliche Gestaltung und Ausstattung des Aufbereitungsraumes sollen sich nach den aktuellen hygienischen Empfehlungen der Kommission für Krankenhaushygiene und Infektionsprävention des Robert Koch-Instituts richten.

Starker Konsens

\subsubsection{Endoskopieeinheit: Raumausstattung von Aufwach-} bereich, Wartebereich und Empfangsbereich

\section{Empfehlung:}

Der Aufwachbereich soll ein definierter Bereich sein, der entsprechend der S3 LL-Sedierung ausgestattet ist, um eine kontinuierliche Überwachung der Patienten zu gewährleisten.

\section{Starker Konsens}

\section{Empfehlung:}

Bei Neuplanung sollte der Wartebereich vom Empfangsbereich einsehbar sein

Starker Konsens

\section{Empfehlung:}

Der Empfangsbereich kann in Form einer Tresens oder eines Büros geplant werden. Dieser soll mit einem zentral vernetzten EDV Arbeitsplatz entsprechend des Patientenaufkommens ausgestattet sein.

Starker Konsens

\subsubsection{Endoskopieeinheit: Weitere Räume \\ Empfehlung:}

Die Größe eines Raumes für Gerätelagerung und Material sollte abhängig von der Größe der Endoskopieeinheit bemessen werden. Starker Konsens

\section{Empfehlung:}

Eine extra Personaltoilette mit hygienischem Händewaschplatz soll getrennt von den Patiententoiletten zu jeder Endoskopieeinheit gehören.

Starker Konsens

\section{Empfehlung:}

Für Patienten- und Angehörigengespräche soll ein geschlossener Raum genutzt werden. Bei Neuplanung sollte ein Besprechungsraum bei größeren Endoskopieeinheiten mit hohem Aufkommen an ambulanten Patienten eingeplant werden.

Konsens

\subsection{Personelle Ausstattung in der gastroenterologischen Endoskopie \\ Empfehlung:}

Für eine belastbare Kalkulation der personellen Ausstattung sollte eine Prozessmessung durchgeführt werden. Auch die „Arbeitsplatzmethode" kann angewendet werden.

Starker Konsens

\subsubsection{Prozessmessung \\ Empfehlung:}

Für die Ermittlung eines angemessenen Stellenplans durch Prozessmessung in der gastroenterologischen Endoskopie sollen eine Reihe von Informationen herangezogen werden:

a) Anzahl und Art der endoskopischen Leistungen pro Jahr

b) Zeitaufwand für die endoskopischen Leistungen (s. Kap. 5.1: Befunddokumentation Zeiterfassung)

c) Zeitaufwand für Tätigkeiten außerhalb des Untersuchungsprozesses

d) Zeitaufwand für qualifizierende Maßnahmen

e) Ausfallzeiten der Mitarbeiter

Starker Konsens ( $\bullet$ Tab. 2, 3)

\subsubsection{Arbeitsplatzmethode Empfehlung:}

Arbeitsplatzmethode: Ein Endoskopiearbeitsraum mit Vollzeitbetrieb soll mit 3 Arbeitskräften besetzt sein: dem endoskopierenden Arzt, der assistierenden Pflegekraft sowie der qualifizierten Person, die die Sedierung überwacht. Ferner soll mindestens eine Person für den Aufwachbereich und sollte eine weitere (anteilig) für die Aufbereitung kalkuliert werden. Ausfallzeiten sollen berücksichtig werden.

Starker Konsens

\subsection{Definition eines Standards für endoskopische Prozeduren für Endoskopiefachberufe 2.4.1 Pflegestandards als Instrumente des Qualitätsmanagements (QM)}

2.4.2 Strukturqualität für endoskopiespezifische Standards 2.4.2.1 Ausbildung und Kompetenzen von Endoskopiepersonal Empfehlung:

Der Arbeitgeber soll die entsprechende Anzahl von qualifiziertem Personal in der Endoskopie zur Verfügung stellen, um eine 
Tab. 2 Definitionen der zu dokumentierenden Untersuchungszeitpunkte.

\begin{tabular}{|ll|}
$\begin{array}{l}\text { Untersuchungs- } \\
\text { zeitpunkt }\end{array}$ & Definition \\
\hline $\begin{array}{l}\text { E1 Patient im } \\
\text { Untersuchungsraum }\end{array}$ & $\begin{array}{l}\text { Patient kommt im Untersuchungsraum an } \\
\text { (Anm.: aus Gründen der Patientensicherheit } \\
\text { und des Haftungsrechtes sollte dieser Zeit- } \\
\text { punkt dokumentiert werden) }\end{array}$ \\
\hline E2 Beginn Endoskopie & $\begin{array}{l}\text { Pflicht: Zeitpunkt, an dem das Gerät in die } \\
\text { Körperöffnung des Patienten eingeführt wird } \\
\text { (für die erste erbrachte Leistung) }\end{array}$ \\
\hline $\begin{array}{l}\text { E3 Beginn Rückzug } \\
\text { des Endoskops }\end{array}$ & $\begin{array}{l}\text { Pflicht: Zur Qualitätssicherung der Koloskopie } \\
\text { laut Leitlinie verpflichtend }\end{array}$ \\
\hline E4 Ende Endoskopie & $\begin{array}{l}\text { Pflicht: Zeitpunkt, an dem das Gerät wieder } \\
\text { aus der Körperöffnung des Patienten entfernt } \\
\text { wurde (für die letzte erbrachte Leistung) }\end{array}$ \\
\hline E5 Patient verlässt & $\begin{array}{l}\text { Patient verlässt Untersuchungsraum (Anm.: } \\
\text { aus Gründen der Patientensicherheit und des } \\
\text { Haftungsrechtes sollte dieser Zeitpunkt do- } \\
\text { Untersuchungsraumentiert werden) }\end{array}$ \\
\hline E6 Patient verlässt & $\begin{array}{l}\text { Patient verlässt Aufwachraum } \\
\text { (Anm.: aus Gründen der Patientensicherheit } \\
\text { und des Haftungsrechtes sollte dieser Zeit- } \\
\text { punkt dokumentiert werden) }\end{array}$ \\
\hline Aufwachraum &
\end{tabular}

Tab. 3 Zu dokumentierendes Personal an den jeweiligen Zeitpunkten.

\begin{tabular}{|l|l|}
\hline Personalbereich & Personalfunktion \\
\hline ärztlicher Dienst & untersuchender Arzt \\
\hline & ärztliche Assistenz 1 \\
\hline & ärztliche Assistenz 2 \\
\hline & sedierender Arzt \\
\hline auszubildender Arzt & Pflege 1 \\
\hline Funktionsdienst & Pflege 2 \\
\hline & Pflege 3 \\
\hline Anästhesie & auszubildende Pflegekraft \\
\hline & Anästhesiearzt \\
\hline & Anästhesiepflege \\
\hline
\end{tabular}

sichere Versorgung der Patienten zu gewährleisten, und Strukturen für dessen kontinuierliche Fort- und Weiterbildung schaffen.

Starker Konsens

\section{Empfehlung:}

Das Endoskopiepersonal soll sich kontinuierlich fortbilden, um eine evidenzbasierte und sichere Patientenversorgung durchführen zu können.

Starker Konsens

\section{Empfehlung:}

In Kliniken und Arztpraxen soll das Endoskopiepersonal über eine fachspezifische Qualifikation und über entsprechende Kompetenzen verfügen, die für die Aufgaben in der Endoskopie notwendig sind.

Starker Konsens

\section{Empfehlung:}

In Endoskopieabteilungen eines Krankenhauses mit endoskopischtherapeutischen Eingriffen soll ein angemessener Anteil (z. B. 50\%) des Endoskopiepersonals eine Fachweiterbildung besitzen. Konsens

\section{Empfehlung:}

Es können erweiterte Aufgaben an endoskopisches Fachpersonal delegiert werden, wenn dieses zuvor im dafür erforderlichen Umfang erweitertes Fachwissen und die dazu notwendigen Fähigkeiten und Fertigkeiten erworben hat.

Starker Konsens

\subsubsection{Anzahl des Personals}

\section{Empfehlung:}

Neben dem Untersucher und seiner direkten Assistenz soll bei endoskopischen Eingriffen unter Sedierung eine entsprechend geschulte und erfahrene Person anwesend sein, die die Sedierung und Überwachung des Patienten übernimmt.

Bei aufwendigen Interventionen und Eingriffen unter sterilen Kautelen soll zusätzliches Personal im Sinne eines Springers einkalkuliert werden.

Die Überwachung des Patienten in der Aufwachphase soll kontinuierlich durch entsprechend geschultes und qualifiziertes Personal gewährleistet sein.

Starker Konsens

\subsubsection{Räumlich-apparative Voraussetzungen}

\section{Empfehlung:}

Endoskopische Eingriffe sollen in dafür eingerichteten Räumlichkeiten durchgeführt werden, die alle Apparate und Instrumente für den Eingriff selbst, die Patientenbetreuung, Sedierung und Überwachung bereithalten. Endoskopiker und Endoskopiepersonal sollten aufgrund ihrer Expertise in die Planung von Endoskopieeinheiten einbezogen werden.

Starker Konsens

\subsubsection{Prozessqualität für endoskopiespezifische Standards Empfehlung:}

Das Endoskopiepersonal soll den Patienten im Rahmen von endoskopischen Eingriffen ganzheitlich betreuen, um Sicherheit und Zufriedenheit des Patienten zu gewährleisten und um mögliche Komplikationen vorzubeugen.

Starker Konsens

\section{Empfehlung:}

Die individuelle Einschätzung des Patienten und seiner Risiken soll vor dem Eingriff gemeinsam durch den endoskopierenden Arzt und die pflegerische Assistenz erfolgen, um eine umfassende Betreuung des Patienten vor, während und nach endoskopischen Eingriffen zu ermöglichen, Komplikationen vorbeugen zu können und um gemeinsam mit dem Untersucher entscheiden zu können, wer die Sedierung und Überwachung während des Eingriffs führt. Starker Konsens

\section{Empfehlung:}

Die Assistenz im Rahmen endoskopischer Eingriffe soll durch qualifiziertes Personal erfolgen.

Konsens

\subsubsection{Ergebnisqualität für endoskopiespezifische \\ Standards}

\section{Statement:}

Die Ergebnisqualität in der Endoskopie ist als gemeinsame Leistung von Medizin und Pflege betrachtet werden, da die Mehrheit der Leistungen gemeinsam erbracht wird.

Starker Konsens 


\subsubsection{Zusammenfassung}

\section{Kap. 3 Prozessqualität - Patientenvorbereitung}

Kap. 3.1 Aufklärung endoskopischer Eingriffe

3.1.1 Aushändigung der Patientenaufklärung an den Patienten

\section{Empfehlung:}

Dem Patienten soll die Aushändigung einer Kopie des vollständig vom aufklärenden Arzt und vom aufgeklärten Patienten ausgefüllten und unterzeichneten Aufklärungsdokuments angeboten werden. Aus juristischer Sicht soll sowohl die Aushändigung der Aufklärung, als auch ein etwaiger Verzicht hierauf in der Patientenakte dokumentiert sowie ggf. vom Patienten quittiert werden. Sofern der Patient auf die Aushändigung ausdrücklich verzichtet, sollte der Patient aus rechtlicher Sicht darauf hingewiesen werden, dass bei Verlangen die Aushändigung von Abschriften auch zu einem späteren Zeitpunkt erfolgen kann.

Starker Konsens

\subsubsection{Arten der Aufklärung}

Die Diagnoseaufklärung

\section{Statement:}

Gegenstand der Aufklärungspflicht des Arztes ist es auch, dem Patienten den medizinischen Befund mitzuteilen.

Konsens

\section{Die Behandlungsaufklärung}

\section{Empfehlung:}

Die Behandlungsaufklärung soll die Art der konkret vorgesehenen endoskopischen Untersuchung bzw. des Eingriffs und auch den Hinweis auf bereits vorhersehbare Erweiterungen des Eingriffs und möglicherweise erforderliche Folgeeingriffe oder Nachoperationen umfassen. Zudem soll über Behandlungsalternativen und über Konsequenzen der Nichtbehandlung aufgeklärt werden. Konsens

\section{Empfehlung:}

Bei Durchführung eines gleichartigen Eingriffes kann eine zuvor durchgeführte Aufklärung über 2-3 Monate ihre Gültigkeit behalten, sofern sich die Risikoeinschätzung nicht erheblich geändert hat. Vor dem erneuten Eingriff soll in jedem Fall ein Patientengespräch mit der Erfassung des aktuellen Wissensstandes erfolgen und schriftlich dokumentiert werden.

Konsens

\section{Empfehlung:}

Der ausdrückliche Wunsch des Patienten auf Nichtbehandlung soll schriftlich niedergelegt und vom Patienten oder von Zeugen des Gesprächs gegengezeichnet werden.

Konsens

\section{Die Risikoaufklärung}

\section{Empfehlung:}

Die Auswahl der aufklärungsbedürftigen Risiken soll sich nicht nach der prozentualen Häufigkeit der Komplikationen richten. Es soll grundsätzlich unabhängig von deren prozentualer Häufigkeit über alle typischen Risiken eines endoskopischen Eingriffs aufgeklärt werden, deren Realisierung erhebliche Auswirkungen auf die Gesundheit und das Leben des Patienten haben kann. Konsens
Die Sicherungsaufklärung

\section{Empfehlung:}

Es soll eine Sicherungsaufklärung über das korrekte Verhalten nach Sedierung und Entlassung aus der ambulanten Therapie erfolgen. Diese soll dem Patienten mündlich erklärt und schriftlich dokumentiert werden.

Konsens

\section{Empfehlung:}

Bei einem Patienten, der sich zu einem ambulanten Eingriff mit Sedierung vorstellt, sollte die Abholung durch eine Begleitperson sichergestellt sein.

Mehrheitliche Zustimmung

\subsubsection{Das Aufklärungsgespräch \\ Empfehlung:}

Das Aufklärungsgespräch soll durch einen fachkundigen Arzt/ Ärztin im Rahmen eines mündlichen für den Patienten verständlichen Gespräches mit dem Patienten durchgeführt werden. Die Aufklärung und Einwilligung sollen in der Patientenakte dokumentiert werden. Auch der Verzicht auf eine Aufklärung soll in der Patientenakte dokumentiert werden. Im Falle von Sprachbarrieren soll ein Übersetzer hinzugezogen werden. Starker Konsens

\subsubsection{Zeitpunkt der Aufklärung Empfehlung:}

Bei größeren therapeutischen endoskopischen Eingriffen bzw. schwerwiegenden Eingriffen mit erhöhtem Risiko soll ein Zeitraum von mindestens 24 Stunden gewahrt werden.

Konsens

\subsubsection{Inhalt des Aufklärungsgespräches Empfehlung:}

Das Aufklärungsgespräch soll sämtliche typischen Risiken der intendierten endoskopischen Untersuchung/Intervention umfassen. Somit muss stets über Art und Schwere des Eingriffs und die möglichen Folgen aufgeklärt werden.

Konsens

\subsubsection{Aufklärungsadressat \\ Empfehlung:}

Bei der Person des Aufklärungspflichtigen soll grundsätzlich der Patient aufgeklärt werden. Besonderheiten bestehen hier bei Minderjährigen und ggf. psychisch kranken bzw. willensunfähigen Personen.

Starker Konsens

\subsection{Endoskopische Prozeduren bei erhöhtem}

Blutungsrisiko

\subsubsection{Laborchemie vor Endoskopie}

Empfehlung:

Ein Routinescreening hinsichtlich einer Koagulopathie vor einem endoskopischen Eingriff mit niedrigem Blutungsrisiko (Bestimmung von Prothrombinzeit [Quick/INR], partieller Thromboplastinzeit, Thrombozyten oder Blutungszeit) sollte in Abwesenheit einer klinischen Anamnese für ein Blutungsereignis, einer Risikoerkrankung oder eines klinischen Verdachtes nicht vorgenommen werden. Ebenso sollte keine Routinetestung bzgl. einer Anämie, Nieren-, Leberfunktionsstörung oder anderer Serumparameter ohne eine klinische Anamnese erfolgen.

Starker Konsens 


\section{Statement:}

Zur Labordiagnostik vor Eingriffen mit hohem Blutungsrisiko kann aufgrund mangelnder Evidenz keine eindeutige Empfehlung abgegeben werden.

Starker Konsens

\section{Empfehlung:}

Eine gerinnungsphysiologische Diagnostik vor dem endoskopischen Eingriff soll bei Vorliegen von anamnestischen Faktoren und Vorerkrankungen erfolgen, die auf ein erhöhtes Blutungsrisiko hinweisen.

Starker Konsens

\subsubsection{Blutungsrisiko endoskopischer Eingriffe Empfehlung:}

Um eine (Nutzen-)Risiko-Abwägung für den Patienten treffen zu können, soll eine Einschätzung des Blutungsrisikos des jeweiligen endoskopischen Eingriffes in Eingriffe mit niedrigem Blutungsrisiko und Eingriffe mit hohem Blutungsrisiko erfolgen ( $\bullet$ Tab. 4). Starker Konsens

Tab. 4 Blutungsrisiko endoskopischer Eingriffe.

\begin{tabular}{ll} 
niedriges Blutungsrisiko & hohes Blutungsrisiko \\
\hline - diagnostische Endoskopie mit/ & - Polypektomie > $10 \mathrm{~mm}$ \\
ohne Biopsie & - EMR/ESD \\
- Gastroskopie & - ERCP mit EST oder Ballondilatation \\
- Kolonoskopie & $>10 \mathrm{~mm}$ \\
- Ballonenteroskopie & - EUS + Feinnadelpunktion zystischer \\
- Kapsel & Läsionen \\
- Polypektomie im Kolon<10 mm & - endoskopische Zystendrainage \\
- ERCP ohne EST mit/ohne Stent & - endosonografisch gestützte Therapie \\
- EUS mit Feinnadelpunktion soli- & - Ballondilatation/Bougierung \\
der Läsionen & - Varizentherapie (Ligatur, \\
- enterale Stentimplantation & Sklerosierung) \\
ohne Dilatation & - PEG \\
- APC von GI Blutungen & - diagnostische Laparoskopie \\
- Thermokoagulation (BARRX) & mit Organbiopsie \\
& - PTC/PTCD
\end{tabular}

Diagnostische Endoskopie mit Biopsie

\section{Empfehlung:}

Die diagnostische Endoskopie mit Zangenbiopsie soll zu den Untersuchungen mit niedrigem Blutungsrisiko gruppiert werden.

Starker Konsens

Endosonografie mit Punktion

\section{Empfehlung:}

Die rein diagnostische EUS ohne und mit Feinnadelbiopsie solider Läsionen sollte zu den Untersuchungen mit geringem Blutungsrisiko kategorisiert werden. Die EUS Feinnadelpunktion zystischer Läsionen und die EUS gesteuerten therapeutischen Verfahren sollen davon abweichend als Eingriff mit hohem Blutungsrisiko bewertet werden.

Starker Konsens

Endoskopische Abtragung von Kolonpolypen

Empfehlung:

Die endoskopische Abtragung von Kolonpolypen unter $10 \mathrm{~mm}$ sollte als Eingriff mit niedrigem Blutungsrisiko bewerten. Die endoskopische Abtragung von Kolonpolypen $\geq 10 \mathrm{~mm}$ sollte als Eingriff mit hohem Blutungsrisiko bewertet werden.

Starker Konsens
Endoskopische Mukosaresektion/endoskopische Submukosadissektion

\section{Empfehlung:}

Die EMR und ESD sollen als Eingriff mit hohem Blutungsrisiko bewertet werden.

Starker Konsens

Endoskopisch biliäre oder pankreatische Sphinkterotomie/Ballondilatation

\section{Empfehlung:}

Die endoskopische biliäre oder pankreatische Sphinkterotomie sowie die großvolumige Ballondilatation (>10 mm) der Sphinkteren sollen zu den Eingriffen mit höhem Blutungsrisiko kategorisiert werden. Die Ballondilatation $<10 \mathrm{~mm}$ kann als Eingriff mit niedrigem Blutungsrisiko gelten.

Starker Konsens

\section{Endoskopische Stenosebehandlung: Dilatation/Bougierung}

\section{Empfehlung:}

Die endoskopische Behandlung von Stenosen im GI-Trakt durch Bougierung und Ballondilatation kann als Eingriff mit hohem Blutungsrisiko bewertet werden.

Starker Konsens

Endoskopische Stenosebehandlung: Selbst expandierende Metallstents

\section{Empfehlung:}

Die Einlage von selbst expandierenden Metallstents im GI Trakt sollte als Eingriff mit niedrigem Blutungsrisiko bewertet werden. Starker Konsens

\section{PEG}

\section{Empfehlung:}

Die Anlage einer perkutanen Gastroenterostomie (PEG) sollte als Eingriff mit hohem Blutungsrisiko bewertet werden.

Starker Konsens

Endoskopische Varizenbehandlung

\section{Empfehlung:}

Die endoskopische Varizenligatur und Varizensklerosierung soll als Eingriff mit hohem Blutungsrisiko bewertet werden.

Starker Konsens

Thermoablative Verfahren

Empfehlung:

Thermoablative Verfahren sollten als Interventionen mit niedrigem Blutungsrisiko bewertet werden.

Konsens

PTC/PTCD

Empfehlung:

Die PTC und insbesondere die PTCD-Anlage soll als Eingriff mit hohem Blutungsrisiko bewertet werden.

Starker Konsens

Diagnostische Laparoskopie

Empfehlung:

Die diagnostische Laparoskopie mit Organbiopsie soll als Eingriff mit erhöhtem Blutungsrisiko gelten.

Starker Konsens 


\subsubsection{Medikation mit Thrombozytenaggregationshem-} mern, Heparinen, oralen Antikoagulantien (Vitamin K-Antagonisten) und direkten oralen Antikoagulantien (DOAK)

\section{Empfehlung:}

Bei geplanten endoskopischen Eingriffen unter Medikation mit Thrombozytenaggregationshemmern und/oder oralen Antikoagulantien soll das individuelle Blutungsrisiko des Patienten unter Berücksichtigung des endoskopischen Eingriffes und der bestehenden Medikation gegen das individuelle Risiko für eine thrombembolische Komplikation durch ein Pausieren der Medikation abgewogen werden. Dies gilt auch für die Wiederaufnahme der Medikation ( $\bullet$ Tab.5 - 8). Im Einzelfall sollte das Vorgehen mit dem behandelnden Kardiologen/Gerinnungsspezialisten abgestimmt werden. Starker Konsens

Tab.5 Klassifizierung des Thrombembolierisikos.

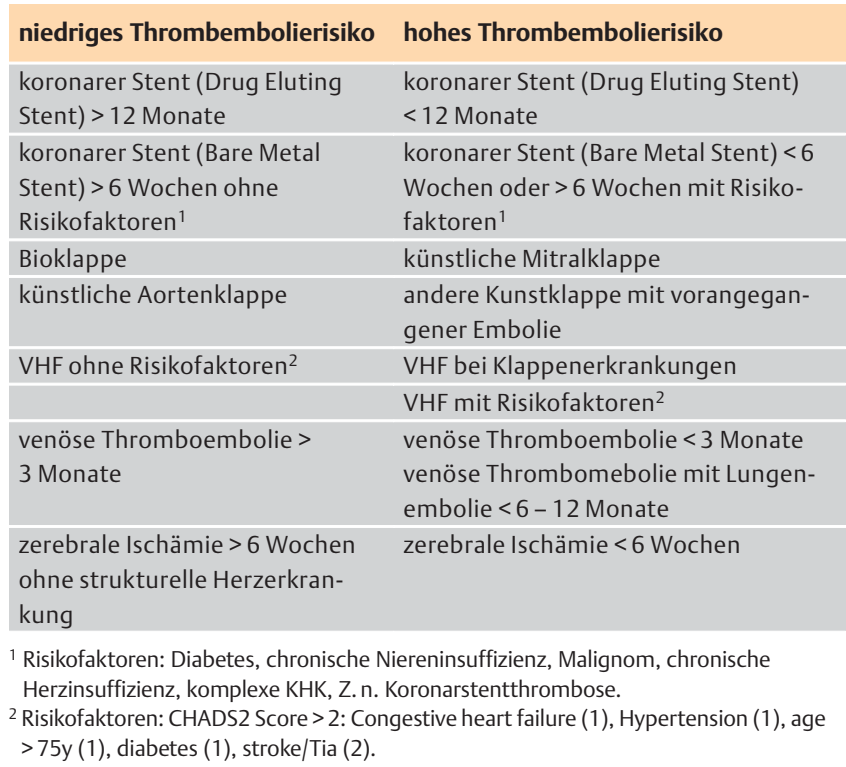

Tab.6 Antikoagulantien: Vorgehen bei Pausieren und Wiedereinnahme.

\begin{tabular}{|lll|}
\hline & $\begin{array}{l}\text { pausieren vor } \\
\text { dem Eingriff }\end{array}$ & $\begin{array}{l}\text { früheste Wiederein- } \\
\text { nahme nach der endo- } \\
\text { skopischen Intervention }\end{array}$ \\
\hline $\begin{array}{l}\text { ASS, Clopidogrel, } \\
\text { Ticlopidin, Ticagrelor }\end{array}$ & 5 Tage & 24 Stunden \\
\hline $\begin{array}{l}\text { Prasugrel } \\
\text { niedermolekulares } \\
\text { Heparin (prophyl- } \\
\text { aktische Dosierung) }\end{array}$ & 7 Tage & 24 Stunden \\
\hline $\begin{array}{l}\text { niedermolekulares } \\
\text { Heparin (therape- } \\
\text { utische Dosierung) }\end{array}$ & 24 Stunden & $6-12$ Stunden \\
\hline $\begin{array}{l}\text { unfraktioniertes } \\
\text { Heparin iv. }\end{array}$ & 4 Stunden & $2-6$ Stunden \\
\hline $\begin{array}{l}\text { Phenprocoumon } \\
\text { (INR } 1 \text { 1,5) }\end{array}$ & 7 Tage & $12-24$ Stunden \\
\hline $\begin{array}{l}\text { direkte orale AK } \\
\text { (DOAK) }\end{array}$ & mind. $24 \mathrm{~h}^{2}$ & $12-24$ Stunden \\
\hline
\end{tabular}

${ }^{1}$ Abhängig von Blutungsrisiko und jeweiligen Komplikationen des Eingriffs.

2 Berücksichtigung von Halbwertszeit und Elimination der DOAK (Tab. 7).

\section{Starker Konsens}

Tab. 7 Direkte orale Antikoagulantien: Halbwertszeit und Elimination.

\begin{tabular}{|ll|}
\hline DOAK-Substanz & HWZ/Elimination \\
\hline Rivaroxaban & $7-11$ h (Elimination 30\% renal, 70\% hepatisch) \\
\hline Apixanban & $9-14$ h (Elimination $25 \%$ renal, $75 \%$ hepatisch) \\
\hline Dabigatran & $12-14-17$ h (Elimination vorwiegend renal) \\
\hline
\end{tabular}

Tab. 8 Empfehlung zum Vorgehen bei Einnahme von TAH, Vitamin K-Antagonisten oder DOAK in Abhängigkeit von der jeweiligen Risikokonstellation.

\begin{tabular}{|c|c|}
\hline $\begin{array}{l}\text { - niedriges Risiko } \\
\text { Endoskopie } \\
\text { - jedes Risiko } \\
\text { thromb- } \\
\text { embolische Kom- } \\
\text { plikation }\end{array}$ & $\begin{array}{l}\text { - Thrombozytenaggregationshemmung weiterfüh- } \\
\text { ren (ASS, Clopidogrel/Ticlopidin oder Prasugrel) } \\
\text { - Heparin, niedermolekulares Heparin weiterführen } \\
\text { - Vitamin K-Antagonisten weiterführen (INR-Kon- } \\
\text { trolle) } \\
\text { - DOAK weiterführen }\end{array}$ \\
\hline $\begin{array}{l}\text { - hohes Risiko } \\
\text { Endoskopie } \\
\text { - niedriges Risiko } \\
\text { für thrombembo- } \\
\text { lische Komplikati- } \\
\text { on }\end{array}$ & $\begin{array}{l}\text { - ASS weiterführen } \\
\text { - ERCP mit EST, Polypektomie im Kolon, PEG, Va- } \\
\text { rizenligatur, EUS-FNA solider Läsionen, Ballon- } \\
\text { dilatation, Bougierung, Stenteinlage, Thermo- } \\
\text { ablation } \\
\text { - ASS } 5 \text { Tage Pause } \\
\text { - EUS-FNA Zyste, EMR, ESD, POEM } \\
\text { - ADP-Rezeptorantagonist: Pause } \\
\text { - Duale TAH (ADP-Rezeptorantagonist + ASS): } \\
\text { ASS weiter } \\
\text { - Vitamin K-Antagonist: Pause, kein Bridging } \\
\text { - DOAK: Pause, kein Bridging } \\
\text { - LMWH Pause }\end{array}$ \\
\hline $\begin{array}{l}\text { - hohes Risiko } \\
\text { Endoskopie } \\
\text { - hohes Risiko für } \\
\text { thrombemboli- } \\
\text { sche Komplikation }\end{array}$ & $\begin{array}{l}\text { - Eingriff verschieben? } \\
\text { - ASS weiterführen } \\
\text { - duale TAH-Diskussion Kardiologie, Gerinnungs- } \\
\text { physiologie } \\
\text { - Pause Clopidogrel/Ticlopidin } 5 \text { d/Prasugrel } 7 \text { d } \\
\text { - Bridging? } \\
\text { - Eingriff unter dualer TAH unter Aufklärung über } \\
\text { erhöhtes Blutungsrisiko } \\
\text { - Vitamin K-Antagonisten absetzen - Bridging } \\
\text { - DOAK absetzten ggf. Bridging vor Wiedereinsetzen } \\
\text { bei Eingriffen mit höherem Nachblutungsrisiko }\end{array}$ \\
\hline
\end{tabular}

Die Datenlage zum jeweiligen Risiko des einzelnen endoskopischen Eingriffes unter Gerinnungshemmender Medikation wird im Folgenden (Kapitel 3.2.4) detailliert aufgeführt.

\section{Starker Konsens}

\subsubsection{Empfehlungen zur Einnahme von gerinnungshem-} mender Medikation in Abhängigkeit vom Risikoprofil bestimmter endoskopischer Eingriffe

Diagnostische Endoskopie mit Biopsie

\section{Empfehlung:}

Bei Durchführung einer diagnostischen Endoskopie mit Zangenbiopsie soll unabhängig vom thrombembolischen Risiko des Patienten keine Pausierung von TAH oder Antikoagulantien erfolgen. Bei Einnahme von Vitamin K-Antagonisten soll die INR nicht oberhalb der Grenze des therapeutischen Bereiches liegen.

\section{Starker Konsens}

\section{EUS-FNA solider/zystischer Läsionen}

\section{Empfehlung:}

Für eine diagnostische EUS-FNA solider Läsionen soll ASS nicht pausiert werden. Aufgrund fehlender Daten zu ADP-Rezeptorantagonisten sollten diese pausiert bzw. bei erhöhtem thrombembolischen Risiko auf ASS umgestellt werden. 
Für die Punktion zystischer Läsionen sollen ADP-Antagonisten pausiert werden. ASS sollte pausiert werden, wenn es das thrombembolische Risiko des Patienten erlaubt.

Niedermolekulares Heparin, Vitamin K-Antagonisten und direkte orale Antikoagulantien sollen entsprechend der Vorgaben pausiert werden.

Starker Konsens

Polypektomie von Kolonpolypen

\section{Empfehlung:}

Für eine Polypektomie von Kolonpolypen jeder Größe soll ASS nicht pausiert werden.

Bei Abtragung von Kolonpolypen > $10 \mathrm{~mm}$ sollten ADP-Rezeptorantagonisten pausiert werden. Ist dies bei hohem thrombembolischem Risiko nicht möglich sollte eine prophylaktische endoskopische Blutstillung erfolgen.

Zur Abtragung von Kolonpolypen sollten niedermolekulares Heparin, Vitamin K-Antagonisten und direkte orale Antikoagulantien entsprechend der Vorgaben pausiert werden.

Polypen bis $5 \mathrm{~mm}$ können unter Antikoagulation mit Vitamin K-Antagonisten mit einer nachfolgend endoskopischen Blutungsprophylaxe abgetragen werden, die INR sollte in diesen Fällen nicht über dem therapeutischen Bereich liegen.

Starker Konsens

EMR/ESD

Empfehlung:

Bei hohem thrombembolischen Risiko kann der Eingriff unter ASS Monotherapie erfolgen unter Aufklärung über ein erhöhtes Blutungsrisiko.

ADP-Rezeptorantagonisten, Niedermolekulares Heparin, Vitamin K-Antagonisten und direkte orale Antikoagulantien sollten entsprechend der Vorgaben pausiert werden.

Starker Konsens

Endoskopische biliäre oder pankreatische Sphinkterotomie Empfehlung:

Für eine ERCP mit Sphinkterotomie soll ASS nicht pausiert werden. ADP-Rezeptorantagonisten sollten pausiert bzw. bei erhöhtem thromboembolischem Risiko auf ASS umgestellt werden.

Niedermolekulares Heparin, Vitamin K-Antagonisten und direkte orale Antikoagulantien sollten entsprechend der Vorgaben pausiert werden.

Starker Konsens

Endoskopische Stenosebehandlung: Dilatation/Bougierung/Stenting Empfehlung:

Ballondilatation, Bougierung sowie Stenting im GI-Trakt können unter ASS erfolgen.

Für eine Ballondilatation oder Bougierung sollten ADP-Rezeptorantagonisten pausiert bzw. bei erhöhtem thrombembolischen Risiko auf ASS umgestellt werden. Niedermolekulares Heparin, Vitamin K-Antagonisten und direkte orale Antikoagulantien sollten entsprechend der Vorgaben pausiert werden.

Starker Konsens

PEG

Empfehlung:

Für eine PEG-Anlage soll ASS nicht pausiert werden.

ADP-Rezeptorantagonisten sollten pausiert werden. Ist dies bei hohem thromembolischem Risiko nicht möglich kann die PEG-An- lage anhand der vorliegenden Daten unter Aufklärung über ein erhöhtes Blutungsrisiko durchgeführt werden.

Niedermolekulares Heparin, Vitamin K-Antagonisten und direkte orale Antikoagulantien sollten entsprechend der Vorgaben pausiert werden.

Starker Konsens

Endoskopische Varizenbehandlung

Empfehlung:

Eine Ligaturtherapie von Ösophagusvarizen kann unter ASS erfolgen. ADP-Rezeptorantagonisten sollten pausiert bzw. bei erhöhtem thrombembolischen Risiko auf ASS umgestellt werden.

Niedermolekulares Heparin, Vitamin K-Antagonisten und direkte orale Antikoagulantien sollten entsprechend der Vorgaben pausiert werden.

Starker Konsens

Thermoablative Verfahren: Argon-Plasma-Koagulation; Radiofrequenzablation

\section{Empfehlung:}

Thermoablative Verfahren können unter ASS erfolgen, ADP-Rezeptorantagonisten sollten bei geringem thrombembolischem Risiko pausiert bzw. auf ASS umgestellt werden.

Vitamin K-Antagonisten Niedermolekulares Heparin und direkte orale Antikoagulantien sollten entsprechend der Vorgaben pausiert werden.

Starker Konsens

\section{PTC/PTCD}

Empfehlung:

Bei hohem thrombembolischen Risiko kann der Eingriff unter ASS-Monotherapie erfolgen unter Aufklärung über ein erhöhtes Blutungsrisiko.

ADP-Rezeptorantagonisten, Niedermolekulares Heparin, Vitamin K-Antagonisten und direkte orale Antikoagulantien sollten entsprechend der Vorgaben pausiert werden.

Konsens

\section{Diagnostische Laparoskopie}

\section{Empfehlung:}

Bei hohem thrombembolischen Risiko kann der Eingriff unter ASSMonotherapie erfolgen unter Aufklärung über ein erhöhtes Blutungsrisiko. ADP-Rezeptorantagonisten, Niedermolekulares Heparin, Vitamin K-Antagonisten und direkte orale Antikoagulantien sollten entsprechend der Vorgaben pausiert werden.

Konsens

3.2.5. Empfehlung zum Vorgehen bei Patienten mit angeborener oder erworbener nicht medikamentös bedingter Hämostasestörung

\section{Empfehlung:}

Vor planbaren endoskopischen Eingriffen bei Patienten mit angeborener oder erworbener nicht medikamentös bedingter Hämostasestörung sollten die konkreten Maßnahmen zur Blutungsprophylaxe und -therapie in Rücksprache mit einem in der Behandlung von Hämostasestörungen erfahrenen Zentrum unter Berücksichtigung des spezifischen Risikos individuell festgelegt werden.

Starker Konsens 
Von-Willebrand-Syndrom (VWS)

VWS Typ 1

\section{Empfehlung:}

Standardmedikament in der Blutungsprophylaxe und -therapie beim VWS Typ 1 soll 1-Desamino-8-D-Arginin-Vasopressin (DDAVP) sein.

Starker Konsens

\section{Empfehlung:}

Zusätzlich zu DDAVP kann bei Eingriffen im Schleimhautbereich das Antifibrinolytikum Tranexamsäure zur Anwendung kommen. Starker Konsens

VWS Typ 2

\section{Empfehlung:}

Die meisten Patienten mit VWS Typ 2 sollten zur Blutungsprophylaxe und -therapie ein VWF-haltiges (Faktor VIII-)Konzentrat erhalten.

Starker Konsens

VWS Typ 3

\section{Empfehlung:}

Die Blutungsprophylaxe vor endoskopischen Eingriffen soll beim VWS Typ 3 mit einem VWF-haltigen (Faktor VIII-)Konzentrat erfolgen.

Starker Konsens

Erworbenes Von-Willebrand-Syndrom (AVWS)

\section{Empfehlung:}

Bei Patienten mit AVWS sollen vor endoskopischen Eingriffen die Maßnahmen zur Blutungsprophylaxe und -therapie unter sorgfältiger Risiko-Nutzen-Abwägung individuell festgelegt werden. Starker Konsens

\section{Hämophilie A und B}

\section{Empfehlung:}

Vor planbaren endoskopischen Eingriffen sollten die konkreten Maßnahmen zur Blutungsprophylaxe und -therapie mit dem behandelnden Hämophiliezentrum abgesprochen und schriftlich festgehalten werden.

Starker Konsens

\section{Hämophilie A \\ Empfehlung:}

Bei Patienten mit milder Hämophilie A sollte die Blutungsprophylaxe bei Eingriffen mit geringem Blutungsrisiko mit DDAVP (Desmopressin) und Tranexamsäure erfolgen.

\section{Starker Konsens}

\section{Empfehlung:}

Bei Patienten mit mittelschwerer oder schwerer Hämophilie A oder bei Patienten mit milder Hämophilie A, die aufgrund des Blutungsrisikos des Eingriffs oder einer schlechten Wirksamkeit oder Verträglichkeit nicht mit DDAVP behandelt werden können, soll die Blutungsprophylaxe mit einem Faktor VIII-Konzentrat erfolgen. Starker Konsens

\section{Hämophilie B}

\section{Empfehlung:}

Patienten mit Hämophilie B sollen zur Blutungsprophylaxe standardmäßig ein Faktor IX-Konzentrat erhalten, da DDAVP (Desmopressin) bei Patienten mit Hämophilie B nicht wirksam ist. Darü- ber hinaus sollen die gleichen Grundsätze und Empfehlungen wie bei Patienten mit Hämophilie A gelten.

Konsens

Hemmkörperhämophilie

\section{Empfehlung:}

Patienten mit Hemmkörperhämophilie sollen zur Blutungsprophylaxe und -therapie sog. Bypass-Präparate erhalten.

Starker Konsens

Andere angeborene Faktormangelzustände

Faktor VII-Mangel

\section{Empfehlung:}

Bei einer Faktor VII-Restaktivität von $>30 \%$ und unauffälliger Blutungsanamnese können endoskopische Eingriffe mit geringem Blutungsrisiko ohne blutungsprophylaktische Maßnahmen oder mit der alleinigen Gabe von Tranexamsäure durchgeführt werden.

Bei einer Faktor VII-Restaktivität von $<30 \%$ oder bei auffälliger Blutungsanamnese und/oder bei Eingriffen mit hohem Blutungsrisiko sollten zur Blutungsprophylaxe zusätzlich Gerinnungsfaktorkonzentrate verabreicht oder zumindest für den Bedarfsfall bereitgehalten werden.

Starker Konsens

\section{Hepatische Koagulopathie}

\section{Empfehlung:}

Die Beurteilung des Blutungsrisikos bei Patienten mit chronischer Lebererkrankung und hepatischer Koagulopathie sollte primär auf der Grundlage anamnestischer und klinischer Daten erfolgen. Ergänzend können hämostaseologische Parameter wie Blutungszeit, Thrombozytenzahl und plasmatische Gerinnungstests (Quick/INR und APTT) herangezogen werden.

Starker Konsens

\subsection{Antibiotikaprophylaxe}

3.3.1 Antibiotikaprophylaxe in Abhängigkeit vom Patientenrisiko

\section{Empfehlung:}

Eine prophylaktische Antibiotikagabe aufgrund eines erhöhten Risikos für eine Herzklappenendokarditis, bei Vorhandensein von Shunts oder endovaskulären Prothesen etc. sollte nicht empfohlen werden.

Starker Konsens

\section{Empfehlung:}

Patienten, die in der Vergangenheit eine Antibiotikaprophylaxe gut vertragen haben, sollte die aktuelle Evidenzlage in einem aufklärenden Gespräch dargelegt werden. Eine Prophylaxe mit einem Enterokokken-wirksamen Antibiotikum sollte nur erfolgen, wenn der Patient nach Aufklärung dies weiterhin wünscht. Starker Konsens

\section{Empfehlung:}

Bei Patienten mit Leberzirrhose soll im Falle einer gastrointestinalen Blutung unabhängig von einer Endoskopie bereits ab dem Zeitpunkt der Aufnahme eine Antibiotikatherapie erfolgen. Starker Konsens

\section{Empfehlung:}

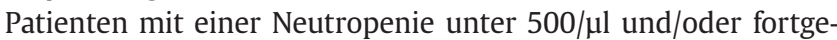
schrittenem Tumorleiden sollten in Absprache mit dem betreu- 
enden Onkologen nur bei therapeutischen Interventionen eine Antibiotikaprophylaxe erhalten. Bei Immunsuppression ohne Neutropenie ist keine Prophylaxe notwendig.

Starker Konsens

\subsubsection{Antibiotikaprophylaxe in Abhängigkeit vom endoskopischen Eingriff \\ Empfehlung:}

Im Rahmen einer ERCP/PTD soll eine Antibiotikaprophylaxe erfolgen, wenn

- bei Gallenwegsobstruktion oder Stentwechsel ohne Cholangitis eine unvollständige Drainage der Gallenwege zu erwarten ist

- eine duktale Endoskopie (+ Therapie) durchgeführt wird

- eine PTD neu angelegt wird

- bei Darstellung des Pankreasgangsystems mit diesem kommunizierende (Pseudo-) Zysten vorliegen

- biliäre Komplikationen nach Lebertransplantation bestehen

- eine Drainage von primär sterilen (Pseudo-)Zysten des Pankreas intendiert ist.

Die Wahl des Antibiotikums soll die zu erwartende Resistenzlage berücksichtigen. Bei vorbestehender Cholangitis oder Zysteninfektion soll die präinterventionell eingeleitete Antibiotikatherapie fortgesetzt werden.

Starker Konsens

\section{Empfehlung:}

Bei einer Endosonografie soll eine Antibiotikaprophylaxe erfolgen, wenn aus einer primär sterilen zystischen Läsion durch Feinnadelaspiration (EUS-FNA) Material gewonnen oder eine transmurale Drainage durchgeführt wird.

Die prophylaktische Antibiotikagabe bei EUS-FNA sollte über 3-5 Tage fortgesetzt werden.

Die Wahl des Antibiotikums soll die zu erwartende Resistenzlage berücksichtigen.

Bei vorbestehender Zysteninfektion soll die präinterventionell eingeleitete Antibiotikatherapie fortgesetzt werden.

Starker Konsens

\section{Empfehlung:}

Bei perkutaner Anlage einer Ernährungssonde in Magen oder Jejunum mit peroralem Durchzug der Sonde soll eine Antibiotikaprophylaxe erfolgen.

Die Wahl des Antibiotikums soll die zu erwartende Resistenzlage berücksichtigen.

Bei MRSA-positiven Patienten soll eine lokale Dekontamination versucht werden ( $\bullet$ Tab. 9 ).

Starker Konsens

\subsection{Patientensicherheit durch Checklisten und Team-Ti-} me-Out (o Tab. 21)

\subsubsection{Standardisiertes Risikoassessment vor dem} endoskopischen Eingriff

\section{Empfehlung:}

Die Patientenvorbereitung sollte mit einem standardisierten Risikoassessment erfolgen, das eingriffs- und sedierungsbedingte Risiken des Patienten ermittelt.

Starker Konsens

\subsubsection{Team-Time-Out vor dem endoskopischen Eingriff Empfehlung:}

Das „Time out“ sollte direkt vor endoskopischen Eingriffen durchgeführt werden, um zu überprüfen, dass der richtige Pa- tient, korrekt vorbereitet, zum richtigen Eingriff aufliegt, das korrekte Equipment funktionsbereit mit dem entsprechenden Personal bereit steht. Individuelle Risiken des Patienten werden kurz genannt.

Starker Konsens

\subsubsection{Standardisiertes Entlassmanagement Empfehlung:}

Die postendoskopische Phase mit Entlassung auf die Station oder nach Hause sollte durch ein standardisiertes Entlassmanagement erfolgen, das eingriffs- und sedierungsrelevante Parameter berücksichtigt

Starker Konsens

\section{Kap. 4 Prozessqualität - Standards für endoskopische Prozeduren}

4.1. Diagnostische Ösophagogastroduodenoskopie

4.1.1 Spezielle Vorbereitung

Empfehlung:

Eine diagnostische ÖGD kann mit Rachenanästhesie oder unter Sedierung erfolgen. Die Durchführung in Sedierung soll immer angeboten werden.

Starker Konsens

\section{Empfehlung:}

Bei endoskopisch suspekten Befunden oder Kontrolluntersuchungen von prämalignen Befunden soll eine ÖGD unter Sedierung empfohlen werden.

Starker Konsens

\subsubsection{Durchführung}

4.1.2.1 Allgemeine Qualitätskriterien

\section{Empfehlung:}

Jede diagnostische ÖGD soll komplett erfolgen, sofern keine Kontraindikationen vorliegen (verbliebene Speisereste im Magen, nicht passierbare Stenose).

Starker Konsens

\section{Empfehlung:}

Neben der Dokumentation von pathologischen Befunden soll eine Bilddokumentation mindestens folgender Landmarken erfolgen: Z-Linie, Antrum, Corpus, Kardia in Inversion, Duodenum Pars II. Starker Konsens

\subsubsection{Gerätetechnik}

\section{Empfehlung:}

Die Art des verwendeten Gastroskops (Durchmesser des Endoskops, Größe des Arbeitskanals, Möglichkeit zur integrierten optischen Kontrastverstärkung mit oder ohne Magnifikation) soll in Abhängigkeit von der Indikation zur Gastroskopie bzw. den vorliegenden Vorbefunden gewählt werden.

Starker Konsens

\section{Empfehlung:}

Zur Diagnostik und Verlaufskontrolle von prämalignen Läsionen in Ösophagus und Magen und Duodenum soll die hochauflösende Videoendoskopie Standard sein.

Starker Konsens 
Tab.9 AB-Prophylaxe bei spezifischen endoskopischen Verfahren.

\begin{tabular}{|c|c|c|c|}
\hline Patient & Prozedur & Ziel der Prophylaxe & periinterventionelle Prophylaxe \\
\hline $\begin{array}{l}\text { Gallenwegsobstruktion/Stent-Wechsel/ } \\
\text { Gallenwege ohne Cholangitis }\end{array}$ & $\begin{array}{l}\text { ERCP mit vollständiger } \\
\text { Drainage }\end{array}$ & Prävention Cholangitis & nicht empfohlen \\
\hline $\begin{array}{l}\text { Gallenwegsobstruktion/Stent-Wechsel/ } \\
\text { Gallenwege ohne Cholangitis }\end{array}$ & $\begin{array}{l}\text { ERCP/PTD mit } \\
\text { unvollständiger Drainage }\end{array}$ & Prävention Cholangitis & $\begin{array}{l}\text { Einzeldosisprophylaxe, Antibiotikatherapie } \\
\text { sollte bis zur vollständigen Drainage fortge- } \\
\text { setzt werden }\end{array}$ \\
\hline mehrfach ERCP mit Z. n. EPT/Stent & ERCP/PTD Stentwechsel & Prävention Cholangitis & $\begin{array}{l}\text { Einzelfallentscheidung zur Einzeldosispro- } \\
\text { phylaxe }\end{array}$ \\
\hline vorbestehende Cholangitis & ERCP/PTD & Prävention Bakteriämie & $\begin{array}{l}\text { Fortsetzung der präinterventionell einge- } \\
\text { leiteten Antibiotikatherapie }\end{array}$ \\
\hline $\begin{array}{l}\text { biliäre Komplikationen nach Lebertrans- } \\
\text { plantation }\end{array}$ & ERCP & Prävention Cholangitis & Einzeldosisprophylaxe \\
\hline duktale Endoskopie (Gallenwege) & ERCP/PTD & Prävention Cholangitis & Einzeldosisprophylaxe \\
\hline intraduktale Lithotrypsie & ERCP/PTD & Prävention Cholangitis & Einzeldosisprophylaxe \\
\hline alle Patienten & PTD-Neuanlage & Prävention Cholangitis & Einzeldosisprophylaxe \\
\hline $\begin{array}{l}\text { mit Pankreasgang kommunizierende } \\
\text { Pankreaszysten oder -pseudozysten }\end{array}$ & ERCP & Prävention Pseudo-/Zysteninfektion & Einzeldosisprophylaxe \\
\hline $\begin{array}{l}\text { Aspiration von pankreatischer Flüssigkeit } \\
\text { (Pseudozyste, Nekrose) mit Pankreas- } \\
\text { gangkommunikation }\end{array}$ & ERCP/EUS-FNA & Prävention Pseudo-/Zysteninfektion & Einzeldosisprophylaxe \\
\hline Aspiration von sterilem Pankreassekret & transmurale Drainage & Prävention Pseudo-/Zysteninfektion & Einzeldosisprophylaxe \\
\hline solide Läsionen im oberen GI Trakt & EUS-FNA & Prävention lokaler Infektion & nicht empfohlen \\
\hline solide Läsionen im unteren GI Trakt & EUS-FNA & Prävention lokaler Infektion & $\begin{array}{l}\text { unzureichende Datenlage für eine } \\
\text { Empfehlung }\end{array}$ \\
\hline zystische Läsionen GI Trakt/Mediastinum & EUS-FNA & Prävention Zysteninfektion & $\begin{array}{l}\text { Einzeldosisprophylaxe (verlängerte Appli- } \\
\text { kation über } 3 \text { - } 5 \text { Tage kann erfolgen) }\end{array}$ \\
\hline alle Patienten & $\begin{array}{l}\text { PEG/PEJ mit peroralem } \\
\text { Durchzug }\end{array}$ & Prävention peristomaler Infektionen & $\begin{array}{l}\text { Einzeldosisprophylaxe MRSA pos.: lokale } \\
\text { Dekontamination (Nase/Rachen) soll } \\
\text { versucht werden }\end{array}$ \\
\hline alle Patienten & Gastropexie-PEG & Prävention peristomaler Infektionen & nicht empfohlen \\
\hline
\end{tabular}

\section{Empfehlung:}

Chromoendoskopie (Indigokarmin, Essigsäure, Plattenephitel: Lugolfärbung) und virtuelle Chromoendoskopie (NBI, FICE, i-Scan) können zur verbesserten Detektion von Dysplasien oder Frühkarzinomen eingesetzt werden.

Starker Konsens

\subsubsection{Biopsie}

\section{Empfehlung:}

Die Biopsie von entzündlichen, präkanzerösen und malignomsuspekten Läsionen soll basierend auf den aktuellen Empfehlungen der Fachgesellschaft/DGVS Leitlinien erfolgen.

Starker Konsens

\section{Empfehlung:}

Zur Entnahme von Mukosabiopsien können Kaltbiopsiezangen mit oder ohne Dorn eingesetzt werden.

Starker Konsens

\section{Empfehlung:}

Die Knopflochbiopsietechnik kann zur Gewinnung von Proben aus submukösen Läsionen eingesetzt werden.

Starker Konsens

\subsubsection{Prozedurabhängige Nachsorge Empfehlung:}

Bei Rachenanästhesie soll eine Nahrungskarenz bis zum vollständigen Abklingen der örtlichen Betäubung eingehalten werden. Starker Konsens

\subsubsection{Komplikationen}

4.1.5 Spezifische Qualitätsindikatoren Diagnostische ÖGD (o Tab. 10)

\section{Tab. 10}

Qualitätsindikatoren
Intraprozedural
Frequenz der Durchführung einer kompletten Ösophagogastroduode-
noskopie
Frequenz der Diagnostik und Verlaufskontrolle von prämalignen Läsio-
nen in Ösophagus, Magen und Duodenum mit hochauflösender Video-
endoskopie
Frequenz der Leitliniengerechten Biopsieentnahme aus entzündlichen,
pathologischen oder prämalignen Läsionen

\subsection{Anlage von Sonden zur enteralen Ernährung}

\subsubsection{Transnasale Sonden}

4.2.1.1 Kontraindikationen zur Ernährung über eine transnasale Sonde

\subsubsection{Vorbereitung \\ Empfehlung:}

Nasoenterische Sonden sollten nach Herstellerangabe vorbereitet werden.

Starker Konsens 


\subsubsection{Durchführung}

\section{Empfehlung:}

Eine nasogastrische Sonde kann in der Regel ohne endoskopische Kontrolle platziert werden. Das Verletzungsrisiko soll durch geeignetes Vorgehen und Vorschub ohne Widerstand gering gehalten werden. Die korrekte Lage soll vor Beginn einer enteralen Ernährung überprüft werden.

Starker Konsens

\section{Empfehlung:}

Transnasale Dünndarmernährungssonden können endoskopisch, radiologisch kontrolliert, aber auch ohne Hilfsmittel platziert werden. Bei Unsicherheit über die korrekte Position soll vor Einleitung einer enteralen Ernährung eine radiologische Lagekontrolle erfolgen. Bei nicht endoskopischer Platzierung soll immer eine radiologische Lagekontrolle erfolgen.

Starker Konsens

\subsubsection{Prozedurabhängige Nachsorge}

Empfehlung:

Sonden zur enteralen Ernährung sollen vor und nach jedem Gebrauch gespült werden.

Starker Konsens

\subsubsection{Komplikationen}

\subsubsection{Perkutane endoskopische Sonden}

\subsubsection{Spezielle Kontraindikationen}

\section{Empfehlung:}

Spezielle Kontraindikationen für eine perkutane, endoskopische Sondenanlage zur Ernährung sind:

- Absolute Kontraindikationen

> allgemeine Kontraindikationen zur enteralen Ernährung

> Unmöglichkeit, die vordere Magenwand (Dünndarmwand) in dauerhaften Kontakt mit der Bauchdecke zu bringen

- fehlende endoskopische Intubierbarkeit des Magens/Intestinums

- floride Peritonitis

- Relative Kontraindikationen

$\checkmark$ negative Diaphanie

- Aszites

- Veränderungen der vorderen Magenwand (intestinalen Wand) durch Tumor, Entzündung oder Ulkus, wenn diese in der Nachbarschaft der Punktionsstelle liegen

> Magenentleerungsstörung

Starker Konsens

\subsubsection{Spezielle Vorbereitung}

\section{Empfehlung:}

Bei malignen Tumoren des Hypopharynx oder des Ösophagus mit kurativem Behandlungsansatz soll die Anlage einer PEG im Direktpunktionsverfahren erfolgen.

Starker Konsens

\section{Empfehlung:}

Die perkutane Punktion bei der PEG-Anlage soll unter sterilen Bedingungen erfolgen.

Starker Konsens

\subsubsection{Durchführung}

\section{Empfehlung:}

Zur Anlage einer perkutanen Ernährungssonde soll nach endoskopischer Intubation des Magens/proximalen Jejunums die kutane Punktionsstelle am Ort der besten Diaphanie gewählt werden. Bei fehlender Diaphanie kann die PEG-Anlage durchgeführt werden, wenn der Aspirationstest negativ ausfällt. Bei der Anlage einer PEJ soll immer eine Probepunktion mit (negativem) Aspirationstest erfolgen. Das weitere Vorgehen bei der Anlage soll Maßnahmen zur Vermeidung von Infektionen und Gewebeschädigung beachten.

Starker Konsens

\subsubsection{Prozedurabhängige Nachsorge}

\section{Empfehlung:}

Eine perkutan platzierte Sonde kann ab dem Tag der Anlage zur Ernährung genutzt werden. In den ersten 7 Tagen nach Anlage sollte ein täglicher Verbandswechsel mit Inspektion und Überprüfung der korrekten Lage erfolgen.

Starker Konsens

\section{Empfehlung:}

Eine PEG/PEJ ohne Gastropexie soll nicht vor Ausbildung einer stabilen Verbindung zwischen Bauchdecke und Magen (Jejunum) entfernt werden. Sonden mit innerer Halteplatte sollten endoskopisch geborgen werden.

Starker Konsens

\section{Empfehlung:}

Bei Sonden, die innen von einem Ballon gehalten werden, sollte dieser Ballon regelmäßig unter Überprüfung des zuvor instillierten Volumens entleert werden, um rechtzeitig Leckagen zu bemerken.

Starker Konsens

\subsubsection{Komplikationen}

\subsection{Endoskopische Varizenbehandlung}

4.3.1 Endoskopisches Varizenscreening

\section{Empfehlung:}

Ein endoskopisches Varizenscreening soll bei jedem Patienten mit einer neu diagnostizierten Leberzirrhose erfolgen.

Eine Wiederholungsendoskopie soll im Falle einer kompensierten Leberzirrhose im Child-Pugh-Stadium A nach 2 Jahren, im Falle einer fortgeschrittenen Zirrhose (Child-Pugh-Stadium B und C) nach einem Jahr durchgeführt werden.

Starker Konsens

\subsubsection{Elektive endoskopische Varizenbehandlung \\ 4.3.2.1 Endoskopische Primärprophylaxe \\ Ösophagusvarizen: Endoskopische Primärprophylaxe \\ Empfehlung:}

Bei Vorliegen von Ösophagusvarizen soll eine Primärprophylaxe erfolgen. Diese kann endoskopisch oder medikamentös erfolgen. Im Falle einer endoskopischen Primärprophylaxe soll eine endoskopische Varizenligatur (EVL) bis zur Varizeneradikation durchgeführt werden.

Starker Konsens

\section{Gastrische Varizen: Endoskopische Primärprophylaxe} Empfehlung:

Die Injektion von Gewebekleber n-Butyl-2-Cyanoacrylat kann bei Vorliegen großer oder unter Therapie der Ösophagusvarizen 
zunehmender gastrischer Varizen mit Blutungsbereitschaftszeichen in der Primärprophylaxe erwogen werden.

Starker Konsens

\subsubsection{Endoskopische Sekundärprophylaxe}

Ösophagusvarizen: Endoskopische Sekundärprophylaxe

\section{Empfehlung:}

Die Kombinationstherapie von endoskopischer Bandligatur und Betablocker soll der Standard in der Sekundärprophylaxe sein, im Falle einer Betablockerunverträglichkeit die alleinige endoskopische Ligaturtherapie.

Starker Konsens

\section{Gastrische Varizen: Sekundärprophylaxe}

\section{Empfehlung:}

Die Injektion von n-Butyl-2-Cyanoacrylat kann bei Z.n. Blutung aus gastrischen Varizen in der Sekundärprophylaxe bis zur Varizeneradikation erfolgen ( $\bullet$ Tab. 11).

\begin{tabular}{|c|c|}
\hline \multicolumn{2}{|c|}{ Primärprophylaxe Ösophagusvarizen } \\
\hline Varizen & NSBB (Propranolol/Carvedilol) \\
\hline $\begin{array}{l}\text { Varizen Grad II-III ohne } \\
\text { Risikofaktoren }\end{array}$ & NSBB/EVL bei Unverträglichkeit \\
\hline $\begin{array}{l}\text { Varizen Grad II-III mit } \\
\text { Risikofaktoren }\end{array}$ & NSBB oder EVL \\
\hline \multicolumn{2}{|c|}{ Sekundärprophylaxe Ösophagusvarizen } \\
\hline Varizen mit Z. n. Blutung & $\begin{array}{l}\text { EVL + NSBB (EVL bei NSBB } \\
\text { Unverträglichkeit) }\end{array}$ \\
\hline \multicolumn{2}{|c|}{ Primärprophylaxe gastrische Varizen } \\
\hline GV mit Risikofaktoren & $\begin{array}{l}\text { NSBB (ggf. Injektion von } \\
\text { n-Butyl-2-Cyanoacrylat) }\end{array}$ \\
\hline \multicolumn{2}{|c|}{ Sekundärprophylaxe gastrische Varizen } \\
\hline GVZ.n. Blutung & $\begin{array}{l}\text { NSBB (ggf. Injektion von } \\
\text { n-Butyl-2-Cyanoacrylat) }\end{array}$ \\
\hline \multicolumn{2}{|l|}{ akute Varizenblutung } \\
\hline Öesophagusvarizen & $\begin{array}{l}\text { EVL/Injektion von n-Butyl-2- } \\
\text { Cyanoacrylat, Interimslösung } \\
\text { Blutungsstent, Reserve: TIPS }\end{array}$ \\
\hline gastrische Varizen & $\begin{array}{l}\text { Injektion von n-Butyl-2-Cyano- } \\
\text { acrylat, Reserve: TIPS }\end{array}$ \\
\hline
\end{tabular}

Risikofaktoren: Child-Pugh-Stadium B-C; red spots, red wale signs. NSBB: nicht selektive Betablocker; EVL: endoskopische Varizenligatur; GV: gastrische Varizen.

\section{Starker Konsens}

\subsubsection{Spezielle Kontraindikationen}

4.3.2.4 Durchführung: Elektive endoskopische Varizenbehandlung Endoskopische Varizenligatur

\section{Empfehlung:}

Vor der Intervention sollte eine aktuelle Beurteilung mittels kompletter Oesophagogastroduodenoskopie erfolgt sein. Es sollen Multibandligatoren verwandt werden und die Varizenligatur sollte möglichst distal am ösophagogastralen Übergang begonnen werden.

Starker Konsens

\section{Empfehlung:}

Die Injektion von n-Butyl-2-Cyanoacrylat gemischt mit Lipiodol (Röntgenkontrast) soll streng in die Varize erfolgen.

Starker Konsens
4.3.2.5 Prozedurabhängige Nachsorge: Elektive endoskopische Varizenbehandlung

Endoskopische Varizenligatur

\section{Empfehlung:}

Nach endoskopischer Varizenbehandlung kann eine stationäre Überwachung abhängig von der Intervention und dem individuellen Risikoprofil des Patienten erfolgen.

Starker Konsens

\section{Fortführung der Ligaturtherapie}

\section{Empfehlung:}

Die Ligaturtherapie soll in regelmäßigen Abständen bis zur Eradikation fortgesetzt werden. Im Folgenden sollten regelmäßige endoskopische Kontrollen erfolgen und ggf. eine erneute Ligaturtherapie bei Auftreten von Rezidivvarizen.

\section{Starker Konsens}

\section{Injektion von Gewebekleber}

Empfehlung:

Im Rahmen der Injektion von n-Butyl-2-Cyanoacrylat sollte eine stationäre Überwachung für mindestens eine Nacht erfolgen. Starker Konsens

\subsubsection{Akute Varizenblutung \\ 4.3.3.1 Spezielle Vorbereitung}

\section{Empfehlung:}

Bei der akuten Varizenblutung soll primär die intensivmedizinische Versorgung mit Stabilisierung des Patienten im Vordergrund stehen.

Starker Konsens

\section{Empfehlung:}

Die Endoskopie zur Diagnosesicherung und Therapie soll nach intensivmedizinischer Stabilisierung bei v.a. eine akute Varizenblutung zeitnah erfolgen.

Starker Konsens

\section{Empfehlung:}

Die pharmakologische Therapie mit einem Vasopressinanalogon sollte bereits bei begründetem Verdacht auf akute Varizenblutung noch vor der Endoskopie begonnen und bei Bestätigung der Diagnose für eine Zeitdauer von 3 - 5 Tagen fortgeführt werden. Starker Konsens

\subsubsection{Durchführung \\ Ösophagusvarizen}

Empfehlung:

Die definitive endoskopische Therapie einer akuten Ösophagusvarizenblutung soll bevorzugt in Form einer Ligaturtherapie erfolgen. Alternativ kann eine Sklerotherapie mit n-Butyl-2-Cyanoacrylat erwogen werden.

Die Sklerosierung mit Etoxysklerol/Polidocanol sollte aufgrund der höheren Komplikationsrate nicht angewandt werden.

Starker Konsens

\section{Gastrische Varizen}

\section{Empfehlung:}

Zur endoskopischen Therapie von akuten Blutungen aus gastrischen Varizen soll eine Injektion von n-Butyl-2-Cyanoacrylat in die blutende Varize erfolgen. Im Einzelfall kann hier insbesondere bei gastrischen Varizen Typ I auch eine Gummibandligatur erfolgen. Starker Konsens 
Endoskopische Interimslösungen bei akuter Varizenblutung Empfehlung:

Bei akuter mit den Standardmaßnahmen nicht beherrschbarer Ösophagusvarizenblutung soll eine alternative Therapie erfolgen. Zur endoskopischen Therapie kann die Insertion eines voll gecoverten selbst expandierenden Blutungsstents erfolgen. Alternativ kann eine Einlage einer Ballontamponade erfolgen.

Starker Konsens

\subsubsection{Komplikationen}

\subsubsection{Spezifische Qualitätsindikatoren ( $\bullet$ Tab. 12)}

\section{Tab. 12}

Qualitätsindikatoren
präprozedural
Frequenz der Antibiotikaprophylaxe bei akuter Varizenblutung
Frequenz der Therapie mit einem Vasopressinanalogon bei akuter
Varizenblutung
intraprozedural
Frequenz der endoskopische Gummibandligatur als das Verfahren der
1. Wahl bei der endoskopischen Therapie der akuten Ösophagusvari-
zenblutung
Frequenz der endoskopische Gummibandligatur als das Verfahren der
1. Wahl bei der endoskopischen Primär- und Sekundärprohylaxe von
Ösophagusvarizen
postprozedural
Frequenz der Empfehlung zur Kontrolle und Fortführung der endosko-
pischen Varizenligatur in der Primär- und Sekundärprohylaxe
Frequenz spezifischer Komplikationen nach Varizenligatur und Injektion
von Gewebekleber

\subsection{Endoskopische Therapie nicht variköser Blutungen} 4.4.1 Zeitpunkt der Endoskopie Empfehlung:

Bei jeder klinisch evidenten gastrointestinalen Blutung sollte eine endoskopische Diagnostik und ggf. Therapie stattfinden.

Starker Konsens

\section{Empfehlung:}

Für die obere gastrointestinale Blutung soll eine frühzeitige Endoskopie (innerhalb von 12 - 24 h nach Aufnahme) erfolgen.

Eine schwere obere GI-Blutung sowie eine erhöhte Mortalität sollte bei Vorliegen bestimmter Faktoren wie zusätzliche Komorbiditäten (Herz-, Leber-, Tumorerkrankung), eingeschränkte klinische und laborchemische Parameter ( $\mathrm{HB}<8 \mathrm{~g} / \mathrm{dl}$, INR $<1,5$, Albumin $<3 \mathrm{~g} / \mathrm{dl}$, RR sys $\leq 90 \mathrm{mmHg}$ ) bedacht werden.

Starker Konsens

\section{Empfehlung:}

Für die klinisch evidente untere GI-Blutung sollte eine Koloskopie nach adäquater Vorbereitung erfolgen. Die Dringlichkeit orientiert sich an der klinischen Situation.

Bei v.a. eine anorektale Blutungsquelle sollte eine Proktorektoskopie vorgeschaltet werden.

Starker Konsens

\subsubsection{Spezielle Vorbereitung Empfehlung:}

Bei Kreislaufinstabilität/Schock soll eine intensivmedizinische Betreuung und eine Kreislaufstabilisierung mit Volumensubsti- tution und ggf. Gabe von Blutprodukten vor/während der Endoskopie erfolgen.

Starker Konsens

Prokinetika bei oberer GI-Blutung

\section{Empfehlung:}

250 mg Erythromycin iv. sollte 30 - 60 Minuten vor der Endoskopie einer frischen oberen gastrointestinalen Blutung mit zu erwartenden Blutkoageln im Magen zur Verbesserung der Beurteilbarkeit verabreicht werden.

Starker Konsens

Protononenpumpeninhibitoren vor der Endoskopie bei oberer Gl-Blutung

\section{Empfehlung:}

Bei Verdacht auf akute Ulkusblutung soll die Therapie mit PPI unverzüglich, unabhängig vom Zeitpunkt der Endoskopie, verabreicht werden.

Starker Konsens

\subsubsection{Durchführung}

\subsubsection{Endoskope und Blutstillungstechniken}

\section{Empfehlung:}

Es sollte ein therapeutisches Endoskop mit großlumigem Arbeitskanal verwendet werden, welches eine suffiziente Spülung und Absaugung erlaubt. Der Anschluss einer automatischen Spülvorrichtung sollte möglich sein.

Konsens

\section{Endoskopische Hämostaseverfahren}

\section{Empfehlung:}

Zur endoskopischen Therapie nicht variköser Blutung können die Injektionstherapie mit $\mathrm{NaCl} 0,9 \%$, verdünnter Suprareninlösung oder die Injektion mit Fibrinkleber sowie thermische Koagulationsverfahren und mechanische Verfahren (Hämoclipping) angewandt werden.

Reserveverfahren stellen der Verschluss mit einem Vollwandverschlussclip und die Sprühapplikation von Hämostasespray dar. Die Verwendung von anderen Sklerosierungssubstanzen zur Therapie der nicht varikösen Blutung kann nicht empfohlen werden.

Starker Konsens

\section{Empfehlung:}

Ein adhärentes Koagel sollte zur Risikoeinschätzung und zur Durchführung der weiteren endoskopischen Therapie entfernt werden.

Starker Konsens

4.4.3.2 Endoskopische Hämostaseverfahren in Abhängigkeit von der Blutungsursache

Peptische Ulkusblutung

\section{Empfehlung:}

Bei Vorliegen von Hochrisikostigmata bei peptischer Ulkusblutung soll eine Injektionstherapie durch ein zweites endoskopisches Hämostaseverfahren (Hämoclip/thermische Verfahren) ergänzt werden, um eine Rezidivblutung zu vermeiden.

Bei ausreichender Beurteilung der Blutungsquelle kann primär eine alleinige Hämoclip Applikation oder ein thermisches Verfahren angewandt werden.

Starker Konsens 
Mallory-Weiss-Läsionen

\section{Empfehlung:}

Zur endoskopischen Therapie von Blutungen aus Mallory-WeißLäsionen des Ösophagus sollten eine Injektionstherapie und/ oder mechanische Verfahren wie z. B. ein Hämoclip Wundrandverschluss Anwendung finden.

Starker Konsens

Vaskuläre Malformation

\section{Empfehlung:}

Zur endoskopischen Therapie von Blutungen aus vaskulären Malformationen sollten thermische Verfahren angewandt werden.

Zur endoskopischen Therapie einer GAVE können thermischen Verfahren oder eine endoskopische Gummibandligatur angewandt werden.

Zur endoskopischen Therapie einer Dieulafoy-Läsion können neben thermischen Verfahren auch mechanische Verfahren (z. B. Hämoclip, Ligatur) angewandt werden.

Starker Konsens

Blutung aus GI-Tumoren

\section{Empfehlung:}

Zur endoskopischen Therapie von Blutungen aus gastrointestinalen Tumoren können thermische Verfahren Anwendung finden.

Starker Konsens

\section{Divertikelblutung}

\section{Empfehlung:}

Eine endoskopische Diagnostik und ggf. Therapie sollte bei v.a. Kolondivertikelblutung nach Darmlavage erfolgen. Zur endoskopischen Hämostase einer akuten Divertikelblutung sollten primär Injektionsfahren und mechanische Verfahren (z. B. Hämoclip, Ligatur) angewandt werden.

Starker Konsens

Blutung während/nach endoskopischer Resektion (Post-Polypektomie, EMR, ESD)

\section{Empfehlung:}

Zur endoskopischen Therapie von intraprozeduralen Blutungen während endoskopischer Resektion können Injektionsverfahren, thermische Verfahren und mechanische Verfahren zum Einsatz kommen. Eine Empfehlung für ein bestimmtes Verfahren oder eine Kombination kann basierend auf der aktuellen Datenlage nicht gegeben werden.

Starker Konsens

4.4.3.4 Endoskopische Blutungsprophylaxe nach endoskopischer Resektion

\section{Empfehlung:}

Zur Prophylaxe von frühen Blutungen nach Polypektomie und endoskopischer Resektion können singuläre oder kombinierte Blutstillungstechniken angewandt werden. Die Entscheidung zur Anwendung einer Blutungsprophylaxe kann bei Vorliegen bestimmter Risikofaktoren (Läsionsgröße $>10 \mathrm{~mm}$, Lokalisation z. B. im rechten Kolon, Komorbiditäten) getroffen werden. Starker Konsens

\subsubsection{Prozedurabhängige Nachsorge Empfehlung:}

Im Falle einer Rezidivblutung sollte ein erneuter endoskopischer Therapieversuch erfolgen.

Starker Konsens

\section{Empfehlung:}

Nach erfolgreicher endoskopischer Hämostase kann die generelle Second-look-Endoskopie nicht empfohlen werden.

\section{Starker Konsens}

\section{Empfehlung:}

Bei Blutung aus peptischen Ulzera mit Hochrisikostigmata sollte eine Hochdosis PPI-Therapie für 72 Stunden erfolgen. Im Verlauf sollte eine HP-Testung und ggf. HP-Eradikation erfolgen. Starker Konsens

\subsubsection{Spezifische Qualitätsindikatoren (o Tab. 13)}

Tab. 13

Qualitätsindikatoren
präprozedural
Frequenz der unverzüglichen PPI-Gabe unabhängig vom Zeitpunkt bzw.
vor der Endoskopie bei Verdacht auf akute Ulkusblutung
intraprozedural
Frequenz der genauen Beschreibung und Dokumentation von Lokalisa-
tion und Art der Blutungsquelle
Frequenz der Anwendung eines zweiten endoskopischen Hämostase-
verfahren (Hämoclip/thermische Verfahren) neben der Injektionsthera-
pie bei Vorliegen von Hochrisikostigmata und peptischer Ulkusblutung
postprozedural
Frequenz der Empfehlung zur HP-Testung und Fortführung einer
PPI-Therapie bei Blutung aus peptischen Ulzera

\section{Kap 4.5 Koloskopie}

\subsubsection{Spezielle Kontraindikationen}

\subsubsection{Spezielle Vorbereitung/Voraussetzungen \\ 4.5.2.1 Vorbereitungsqualität}

\section{Empfehlung:}

Durch die Vorbereitung sollen bei der Untersuchung keine oder nur noch minimale Stuhlmengen nachzuweisen sein, die während der Untersuchung durch Spülung und Absaugen zu entfernen sind, um eine optimale Untersuchungsqualität zu gewährleisten.

Starker Konsens

Die Vorbereitungsqualität soll im Untersuchungsbefund dokumentiert werden, auch wenn gute Evidenz für ihre Eignung als Qualitätsparameter fehlt; hierzu kann ein vereinfachter und auf das gesamte Kolon ausgedehnter Boston-Bowel-Preparation-Score verwendet werden.

Konsens

Untersuchungen, die eine schlechte Vorbereitungsqualität (Score 1) im vereinfachten Boston-Bowel-Preparation-Score haben, haben eine niedrigere Adenomdetektionsrate und sollten wiederholt werden, wenn es um Vorsorgeaspekte und Neoplasiedetektion geht. Starker Konsens

\subsubsection{Voraussetzung zur Durchführung (Untersucher) Empfehlung:}

Um ausreichende Erfahrung und Kompetenz in der Durchführung von Koloskopien zu haben, sollte nach einer kumulativen Erfahrung in Aus- und Weiterbildung von 300 Koloskopien - im Einklang mit den derzeitigen Festlegungen - eine Mindestmenge von 200 Koloskopien/Jahr durchgeführt werden.

Konsens 


\section{Statement:}

Die Einhaltung der unten genannten Qualitätsparameter Zökumund Adenomdetektionsrate erscheint wichtiger als starre Fallzahlgrenzen.

Konsens

\subsubsection{Sedierung}

\section{Empfehlung:}

Um eine hohe Akzeptanz der Untersuchung zu erreichen aber auch um die Komplettierungsrate der Untersuchung zu optimieren, soll nach Abwägung von Risiko und Nutzen eine Sedierung bei der Untersuchung angeboten werden.

Starker Konsens

\subsubsection{Durchführung \\ 4.5.3.1 Zeiterfassung \\ Empfehlung:}

Bei einer Koloskopie soll die Zeit, in der das Koloskop unter sorgfältiger Inspektion der Schleimhaut zurückgezogen wird, dokumentiert werden.

Die Zökumrückzugszeit sollte mindestens 6 Minuten betragen. Hierunter fallen nicht die Zeiten für Biopsie und Polypektomie. Starker Konsens

\section{Statement:}

Die zugrunde liegenden Studien sind aber nicht einheitlich, vor allem, was den Einfluss einer höheren Untersuchungszeit einzelner Endoskopiker auf die Adenomdetektionsrate betrifft.

Starker Konsens

4.5.3.2 Parameter zur Erfolgskontrolle der diagnostischen Koloskopie

Empfehlung:

Eine Qualitätskontrolle der Koloskopie soll im Hinblick auf die Detektion von Dickdarmneoplasien im ambulanten und stationären Bereich erfolgen.

Konsens

\section{Vollständigkeit/Zökumintubationsrate}

\section{Empfehlung:}

Eine Zökumintubationsrate von mehr als $90 \%$ soll bei allen intendiert kompletten Koloskopien erreicht werden. Das Erreichen des Zökums soll durch Fotodokumentation des Orificium des Appendix und der Ileokoekalklappe dokumentiert werden.

Starker Konsens

\section{Adenomdetektionsrate}

Empfehlung:

Im Rahmen einer Vorsorgekoloskopie sollen in $\geq 20 \%$ der Untersuchten Adenome detektiert werden. Auch in Kliniken soll die Adenomdetektionsrate (Rate von Patienten mit mindestens einem Adenom) für die intendiert vollständige diagnostische Koloskopie dokumentiert werden.

Starker Konsens

Dies gilt nicht für Überweisungen mit bekannter Diagnose, chronisch entzündliche Darmerkrankungen, Patienten mit Blutungen und Notfallindikationen sowie Patienten mit Zuweisung zur endoskopischen Therapie inklusive Polypektomie aus. Für die Vergleichbarkeit mit der ambulanten Vorsorgekoloskopie ist eine Alterskorrektur wünschenswert.

Konsens

\section{Komplikationsrate als Qualitätsparameter}

\section{Empfehlung:}

Die Komplikationen der Koloskopie sollen im Rahmen der gesetzlichen Vorgabe dokumentiert werden.

Starker Konsens

\section{Statement:}

Die Komplikationsrate ist zwar ein logischer, aber schwer überprüfbarer Qualitätsparameter; bevor Festlegungen über Art, Definition der Komplikation und den Zeitraum der Erfassung nach dem Eingriff allgemeinverbindlich getroffen, in ihrem Aufwand abgeschätzt und Einigkeit über ein unabhängiges Monitoring getroffen sind, ist die Komplikationsrate bei der diagnostischen und therapeutischen Koloskopie nicht zum breiten Einsatz und zum Benchmarking geeignet

Starker Konsens

\subsubsection{Geräte- und Untersuchungstechnik}

\section{Empfehlung:}

Koloskopische Untersuchungen sollen mit hochauflösenden Videoendoskopen durchgeführt werden.

Starker Konsens

\section{Statement:}

Die Anwendung erweiterter Bildgebungsverfahren (z.B. direkte und virtuelle Chromoendoskopie/Zoomendoskopie) stellt in bestimmten Situationen eine Verbesserung dar.

Starker Konsens

\section{Verwendung von $\mathrm{CO} 2$ bei der Koloskopie}

\section{Empfehlung:}

Koloskopien sollten mit CO2-Insufflation durchgeführt werden, da dies den Patientenkomfort durch eine Reduktion der abdominellen Beschwerden nach der Koloskopie erhöht. Wahrscheinliche Vorteile liegen auch bei therapeutischen Eingriffen mit einem erhöhten Risiko für eine Perforation.

Starker Konsens

Gabe spasmolytisch wirksamer Medikamente während oder vor der Koloskopie

\section{Empfehlung:}

Spasmolytisch wirksame Medikamente können in Einzelfällen bei fehlenden Kontraindikationen zur besseren Entfaltung des Kolons gegeben werden.

Starker Konsens

4.5.3.4 Biopsieverhalten und endoskopische Differenzialdiagnose von Kolonneoplasien

\section{Empfehlung:}

Karzinomverdächtige Läsionen sollen biopsiert werden, wenn keine primäre Abtragung geplant ist.

Starker Konsens

Vor Polypektomie sollte eine Biopsie nur dann erfolgen, wenn die Art der Läsion unsicher bleibt (Neoplasie oder Entzündung, Hyperplasie) und das weitere Vorgehen beeinflusst wird (z. B. verschiedene Resektionsverfahren).

Starker Konsens

\section{Statement:}

Es gibt keine ausreichende publizierte Evidenz, dass Biopsien nachfolgende endoskopische Resektionen erschweren.

Starker Konsens 


\section{Statement:}

Der endoskopische Aspekt ist als Gesamtbeurteilung vor einer endoskopischen Polypenresektion wichtig und beinhaltet Aspekte der Morphologie (Verlust der Struktur, Ulzeration/Einsenkung, Vulnerabilität) und des Verhaltens bei der Biopsie (Gewebehärte) und ggf. beim versuchten Hochspritzen (non-lifting sign), auch wenn die Treffsicherheit der jeweiligen Einzelparameter beschränkt ist.

Starker Konsens

\subsubsection{Histologie nach Adenomresektion}

\section{Empfehlung:}

Abgetragene Läsionen sollen geborgen und unter Angabe der Lokalisation zur histologischen Untersuchung eingesandt werden. Gelingt dies im Einzelfall nicht, soll dies im Befund vermerkt werden.

Starker Konsens

\subsubsection{Endoskopische Resektion von Kolonneoplasien}

\section{Empfehlungen:}

Adenome im Kolon und Rektum sollen vollständig entfernt werden.

Bis zu einer Polypengröße von $5 \mathrm{~mm}$ kann die Polypektomie auch ohne Diathermie, bevorzugt mit einer Schlinge oder mit einer Biopsiezange erfolgen.

Starker Konsens

\section{Statement:}

Da die vollständige Abtragung mit Zange nur bis etwa $3 \mathrm{~mm}$ dokumentiert ist, ist bei grenzwertig großen Polypen um 4-5 mm die Kaltabtragung mit einer Schlinge das präferierte Verfahren. Konsens

\section{Empfehlungen:}

Bei einer Polypengröße $>5 \mathrm{~mm}$ soll eine Schlingenabtragung mit oder ohne Unterspritzung unter Verwendung von Diathermiestrom durchgeführt werden. Bei flachen Adenomen sollte die Abtragung in Form einer EMR (endoskopische Mukosaresektion, besser saline-assisted polypectomy) erfolgen.

Klassische sogenannte EMR-Techniken wie die kappenunterstützte „Suck and cut“-Technik und die „Bandligatur“-Technik sollten nur im Rektum angewandt werden.

Starker Konsens

\section{Empfehlung:}

Die endoskopische Submukosadissektion (ESD) ist in westlichen Ländern aufgrund der hohen Komplexität, variablen Erfolgsrate und erhöhten Komplikationsrate keine etablierte Resektionstechnik für kolorektale Läsionen und sollte spezialisierten Zentren vor allem im Rahmen von Studien vorbehalten bleiben.

\section{Starker Konsens}

\section{Empfehlung:}

Die Vollständigkeit der Abtragung soll endoskopisch kontrolliert werden. Diesbezüglich richten sich die Nachsorgeintervalle nach den Empfehlungen der Leitlinie Kolorektales Karzinom der DGVS. Bei Piecemeal-Abtragung sollen im Rahmen der ersten endoskopischen Nachsorge auch Biopsien aus makroskopisch unauffälligen Narbenarealen erfolgen.

Starker Konsens

\subsubsection{Spezifische Qualitätsindikatoren}

(o Tab. 14)

\section{Tab. 14}

Qualitätsindikatoren Koloskopie
präprozedural
siehe allgemeine Qualitätsindikatoren Endoskopie
intraprozedural
Frequenz der Dokumentationsrate der Vorbereitungsqualität
(vereinfachter Boston-Score)
Zoeumintubationsrate (Dokumentation Appendixabgang/Zökumboden
und Ileozoekalklappe)
Frequenz der Dokumentation der Rückzugszeit (im Rahmen der Zeiter-
fassung)
Adenomdetektionsrate (Rate von Patienten mit mind. einem Adenom)
bei Vorsorgevoloskopie oder adäquaten Indikationen
Frequenz der Dokumentation der Vollständigkeit bei Polypenabtragung
(endoskopische Beurteilung)
postprozedural
Frequenz der Vollständigkeit der Einsendung von Präparaten zur histo-
logischen Untersuchung nach Polypektomie oder ggf. Biopsie von nicht
abtragbaren Polypen
sonst siehe allgemeine Qualitätsindikatoren Endoskopie (v. a. Nachsor-
geempfehlungen adaptiert an histologische Befunde)

Kap 4.6 Endoskopische Resektion

4.6.1 Spezielle Vorbereitung

4.6.1.1 Endoskopische Beurteilung vor endoskopischer Resektion Empfehlung:

Vor endoskopischer Resektion soll die Läsion in hochauflösender Videoendoskopietechnologie hinsichtlich Ausdehnung, Oberflächenmuster und Vaskularisierung beurteilt werden.

\section{Starker Konsens}

\section{Empfehlung:}

Additiv kann die Beurteilung durch Chromoendoskopie bzw. virtuelle Chromoendoskopie ergänzt werden.

\section{Starker Konsens}

\section{Empfehlung:}

Eine Klassifikation des mukosalen Musters (Pit-Pattern) und der Mikroarchitektur der mukosalen und submukosalen Gefäße („vessel-pattern“) sowie eine Beurteilung nach der Paris-Klassifikation kann sinnvoll sein.

Starker Konsens

\section{Empfehlung:}

Das Plattenephitelkarzinom des Ösophagus soll in seiner Ausdehnung vor endoskopischer Resektion mittels 1 - 3 \%iger Kalium-Jodid-Lösung (Lugol-Lösung) beurteilt werden.

Konsens

\section{Empfehlung:}

Ein endoskopischer Ultraschall (EUS) soll zum Ausschluss eines fortgeschrittenen T-Stadiums und zur Detektion von Lymphknoten erfolgen.

\section{Starker Konsens}




\subsubsection{Durchführung}

4.6.2.1 Allgemeines

Empfehlung:

Interventionelle endoskopische Resektionen sollen in Sedierung durchgeführt werden. Je nach Invasivität und Zeitumfang kann die Durchführung in Intubationsnarkose erfolgen.

Starker Konsens

Lagerung während der endoskopischen Resektion

Empfehlung:

Endoskopische Resektionsverfahren (ER/ESD) sollten bei nicht intubierten Patienten in der Regel in Linksseitenlage ausgeführt werden. Je nach Lokalisation und Konfiguration der Läsion kann aus Gravitätsgründen eine Umlagerung des Patienten erfolgen.

Starker Konsens

\subsubsection{Gerätetechnik \\ CO2-Insufflation}

Empfehlung:

Zur EMR und ESD sollte CO2 anstelle von Raumluft eingesetzt werden. Bei der endoskopischen peroralen Myotomie (POEM) und bei submukosalen Resektionen und Tunnelungstechniken soll $\mathrm{CO} 2$ verwendet werden.

Starker Konsens

Endoskope

Empfehlung:

Zur Durchführung einer Endoskopischen Resektion sollen Standardendoskope eingesetzt werden. Spezialendoskope mit Elevationseinrichtungen am Arbeitskanal und neue endoskopische Plattformen befinden sich in der Erprobung und sollten innerhalb von Studien eingesetzt werden.

Starker Konsens

\section{HF-Chirurgie}

Empfehlungen:

Zur Durchführung einer endoskopischen Resektion sollen Mikroprozessor-HF-Generatoren mit automatisierter Schneide- und Koagulationsfunktion verwendet werden.

Starker Konsens

Die elektrochirurgische Einstellung soll in Abhängigkeit von der von der Art des Eingriffs, der Expertise des Untersuchers und den Herstellerangaben zum verwendeten Instrumentarium gewählt werden.

Starker Konsens

\section{Injektionslösungen}

Empfehlung:

Zur submukosalen Injektion bei ER soll $\mathrm{NaCl} \mathrm{0,9 \%} \mathrm{Standard} \mathrm{sein,}$ ein Zusatz von Adrenalin kann erfolgen. Alternativ können hochvisköse Injektionslösungen insbesondere zur EMR oder ESD angewandt werden, um eine zeitstabileres submuköses Kissen zu bilden. Ein Zusatz von Farbe zur besseren Visualisierung der Wandschichtung kann erfolgen.

Starker Konsens
4.6.2.3 Endoskopische Resektionstechniken

Endoskopische Mukosaresektion

Empfehlung:

Zur EMR können verschiedene Techniken („inject and cut“, „suck-and-cut”, „band-ligation-EMR“, „zirkumferenzielle CEMR“) zum Einsatz kommen.

Starker Konsens

\section{Statement:}

Die Kappen unterstützte „suck and cut“-Technik und die „Bandligatur“-Technik sind anhand der Datenlage für die Barrett-Neoplasie gleichermaßen effektiv.

Starker Konsens

Endoskopische submukosale Dissektion (ESD)

Empfehlung:

Die ESD-Technik und die Auswahl des Instrumentariums sollen sich nach der Expertise des Untersuchers richten. Basierend auf der aktuellen Datenlage kann keine Empfehlung für eine bestimmte Technik oder Instrumentarium gegeben werden.

Starker Konsens

4.6.2.4 Endoskopische Resektionsverfahren in Abhängigkeit von der Läsion

\section{Empfehlung:}

Ziel der ER soll die vollständige und kurative Resektion der Läsion sein. Die verwendete Technik zur ER soll sich nach der Art, Größe und Lokalisation der GI-Läsion richten.

Starker Konsens

\section{Empfehlung:}

EMR-Verfahren können zur endoskopischen En-bloc-Resektion von GI-Läsionen bis zu einer maximalen Größe von $20 \mathrm{~mm}$ angewandt werden und darüber hinaus zur Piecemeal-Abtragung. Starker Konsens

\subsubsection{Prozedurabhängige Nachsorge \\ Empfehlung:}

Nach endoskopischen Resektionsverfahren sollte in Abhängigkeit von dem individuellen Risikoprofil des Patienten, der Größe und Lokalisation der Läsion sowie der Komplexität und dem Verlauf der Intervention über eine stationäre Nachsorge entschieden werden. Nach ESD soll die Nachsorge grundsätzlich stationär erfolgen.

\section{Statement:}

Bzgl. der Nachsorge nach Resektion neoplastischer Läsionen wird auf die entsprechenden AWMFLL (www.awmf.org/leitlinien/ aktuelle-leitlinien.html) verwiesen.

Konsens

4.7 Endoskopische enterale Dilatation, Bougierung und Stenteinlage in derTherapie von Stenosen in Ösophagus, Magen, Duodenum, Kolon (benigne/maligne) und postoperativen Leckagen

\subsubsection{Spezielle Vorbereitung}

Empfehlung:

Nur GI-Stenosen, die Symptome und/oder eine objektivierbare Passagestörung verursachen, sollen endoskopisch behandelt werden.

Starker Konsens 


\section{Empfehlung:}

Vor Behandlung einer GI-Stenose soll sichergestellt sein, dass keine nachgeschalteten (weiter distal gelegenen) oder vorgeschalteten (weiter proximal gelegenen) Stenosen bestehen.

Bei klinischem v.a. auf weitere Stenosen sollte neben der endoskopischen Untersuchung eine geeignete Bildgebung (CT, MRT Sellink, US Abdomen, Gastrografinkontrast) erfolgen.

Starker Konsens

\subsubsection{Durchführung}

4.7.2.1 Ösophagusstenosen, benigne

\section{Empfehlung:}

Zur endoskopischen Therapie von benignen Stenosen des Ösophagus (peptische Stenosen, Anastomosenstenosen) soll primär eine Bougierung oder Ballondilatation durchgeführt werden. Starker Konsens

\section{Empfehlung:}

Die Auswahl des Verfahrens sollte sich nach der Lage, Länge und Komplexität der Stenose richten.

Starker Konsens

\section{Empfehlung:}

Der initial gewählte Durchmesser der Bougierung bzw. Ballondilatation soll sich an der vorliegenden Weite der Stenose orientieren.

Die Bougierung/Ballondilatation kann stufenweise über 2-3 Stufen (von je 1-1,5mm) erfolgen, bei einfachen Stenosen kann in einer Sitzung auch eine Dilatation über $3 \mathrm{~mm}$ hinaus durchgeführt werden. In ausgewählten Fällen empfiehlt sich eine zwischenzeitige endoskopische Kontrolle.

Starker Konsens

\section{Empfehlung:}

Die Bougierung oder Dilatation soll unter endoskopischer oder bei hochgradiger Stenose ohne sichere endoskopische Drahteinlage unter fluoroskopischer Kontrolle erfolgen.

Starker Konsens

\section{Empfehlung:}

Bei therapierefraktärer benigner Ösophagusstenose kann eine temporäre Stentimplantation erfolgen. Verfügbar sind voll gecoverte SEMS, SEPS und biodegradierbare Stents.

Starker Konsens

\section{Empfehlung:}

Die Stenteinlage kann drahtgeführt unter fluoroskopischer und/ oder endoskopischer Kontrolle erfolgen.

Starker Konsens

\section{Empfehlung:}

Partiell gecoverte selbst expandierende Metallstents sollten wegen der erhöhten Komplikationsrate (Einwachsen, Rezidivstrikturen, erschwerte Stentenfernung) nicht verwendet werden.

Starker Konsens

\section{Empfehlung:}

Zur Rezidivprophylaxe kann eine Injektion von Steroiden in die Stenose erfolgen.

Starker Konsens

\subsubsection{2 Ösophagusstenose, maligne}

\section{Empfehlung:}

In der palliativen Therapie von malignen Stenosen des Ösophagus (Ösophaguskarzinom) und des Magens (Kardiakarzinom) sollte eine endoskopische Therapie (SEMS, Lokalablative Verfahren) erfolgen.

Starker Konsens

\section{Empfehlung:}

Bei gleichzeitiger Radiochemotherapie im palliativen Therapiekonzept kann die Einlage eines SEMS erfolgen.

Starker Konsens

\section{Empfehlung:}

Im neoadjuvanten Therapiekonzept von malignen Stenosen des Ösophagus (Ösophaguskarzinom) und des Magens (Kardiakarzinom) sollte bei anhaltender Dysphagie die Einlage eines SEMS sorgfältig abgewogen werden.

Starker Konsens

\section{Empfehlung:}

In der endoskopischen palliativen Therapie maligner Stenosen des Ösophagus und des gastroösophagealen Überganges sollte die Einlage eines SEMS den lokal ablativen Verfahren vorgezogen werden.

Starker Konsens

\section{Empfehlung:}

Ballondilatation, Bougierung oder lokale Gewebeablation mittels Laser- oder APC-Koagulation können zur Vorbereitung dieser Maßnahme oder bei Unverträglichkeit oder fehlender Platzierbarkeit für einen SEMS eingesetzt werden.

Starker Konsens

\section{Empfehlung:}

Eine Empfehlung für einen bestimmten SEMS-Typ kann auf der Basis der aktuellen Evidenz nicht gegeben werden.

Starker Konsens

\subsubsection{Magenausgangstenose, benigne}

\section{Empfehlung:}

In der endoskopischen Therapie von benignen Magenausgangsstenosen (Pylorus, Duodenum) soll primär eine Ballondilatation durchgeführt werden.

Starker Konsens

\section{Empfehlung:}

Die Ballondilatation soll unter endoskopischer Sicht angepasst an die jeweilige Stenose erfolgen.

Starker Konsens

\section{Empfehlung:}

Begleitend soll eine säurehemmende Therapie und, wenn H. pylori positiv, eine HP-Eradikation erfolgen.

Starker Konsens

\section{Empfehlung:}

Die Implantation eines SEMS soll bei benigner Magenausgangsstenose ausgewählten Einzelfällen vorbehalten bleiben.

Starker Konsens 


\section{Empfehlung:}

Bei Versagen der Dilatationstherapie oder Rezidiven nach initial erfolgreicher Dilatation sollte eine chirurgische Therapie (Gastroenterostomie, distale BII-Magenresektion) durchgeführt werden.

Starker Konsens

\subsubsection{Magenausgangsstenose, maligne \\ Empfehlung:}

Zur Therapie einer malignen Stenose von Magenausgang und/ oder Duodenum soll in Abhängigkeit von der individuellen Prognose des Patienten, dem Lokalbefund und der Präferenz des Patienten zwischen konservativer (Ablaufsonde), endoskopischer Therapie und chirurgischer Therapie (Bypass) entschieden werden.

Starker Konsens

\section{Empfehlung:}

Zur endoskopischen Therapie einer malignen Stenose von Magenausgang und/oder Duodenum kann in der palliativen Situation eine Stentimplantation erfolgen.

Starker Konsens

\subsubsection{Benigne Stenose des unteren GI-Traktes \\ Empfehlung:}

In der endoskopischen Therapie von benignen Stenosen des ileocoecalen Übergangs und des Kolons sollte bei technischer Machbarkeit (gute Erreichbarkeit, kurzstreckige Stenose) eine Ballondilatation durchgeführt werden.

Starker Konsens

\section{Empfehlung:}

Endoskopische Dilatation von entzündlichen Stenosen sollte immer in eine antiinflammatorische Therapie eingebunden werden. Starker Konsens

\section{Empfehlung:}

Zur Rezidivprophylaxe kann eine simultane Steroidinjektion durchgeführt werden.

Starker Konsens

Empfehlung:

Bei rezidivierender oder therapierefraktärer Stenose sollte eine Operation durchgeführt werden.

Starker Konsens

\subsubsection{Maligne Stenose des Kolons}

\section{Empfehlung:}

Zur endoskopischen Therapie einer malignen Stenose des Kolons kann in der Notfallsituation (akute Obstruktion) die Implantation eines selbst expandierenden Metallstents („bridge to surgery“) durchgeführt werden.

Starker Konsens

\section{Empfehlung:}

In der palliativen Situation kann bei Patienten mit hoher Komorbidität, geringer Lebenserwartung und hohem operativen Risiko als Alternative zur chirurgischen Resektion oder zur Stuhldeviation (Bypass, Stoma) eine Stentimplantation erfolgen.

Starker Konsens

\subsubsection{Postoperative Leckage}

\section{Empfehlung:}

Zur endoskopischen Therapie einer postoperativen Leckage am oberen Gastrointestinaltrakt kann die Einlage eines komplett gecoverten Stents oder eine endoskopische Vakuumtherapie erfolgen. Starker Konsens

\section{Empfehlung:}

Zur endoskopischen Therapie einer postoperativen Leckage am unteren Gastrointestinaltrakt kann die endoskopische Vakuumtherapie erfolgen.

Starker Konsens

\section{Empfehlung:}

Bei geeigneten Leckagen (kleiner Durchmesser, keine infizierte Insuffizienzhöhle) im oberen und unteren Gastrointestinaltrakt kann ein Verschluss mit Clipping-Devices erfolgen.

Starker Konsens

\subsubsection{Prozedurabhängige Nachsorge Empfehlung:}

Zum Abschluss jeder interventionellen Stenosetherapie soll eine endoskopische Kontrolle erfolgen, um akute Blutungen oder Perforationen auszuschließen.

Starker Konsens

\section{Empfehlung:}

Wegen der noch zweizeitig möglichen Perforation kann in Abhängigkeit vom Risikoprofil eine Überwachung unter stationären Bedingungen erfolgen.

Starker Konsens

\subsection{Endoskopisch-retrograde Cholangiopankreatiko-} grafie (ERCP)

4.8.1 Spezielle Kontraindikationen

\subsubsection{Spezielle Vorbereitung}

Lagerung während der ERCP

Empfehlung:

Die ERCP sollte bei nicht intubierten Patienten in Bauchlage (oder alternativ in Linksseitenlage) ausgeführt werden.

\section{Starker Konsens}

\section{Empfehlung:}

Die ERCP soll unter sterilen Ausgangsbedingungen für den Instrumentierungstisch erfolgen.

Starker Konsens

\section{CO2-Insufflation}

\section{Empfehlung:}

Die Verwendung von CO2 in der ERCP kann postinterventionelle Schmerzen und die abdominelle Distension reduzieren. Starker Konsens

\section{Empfehlung:}

Die perorale Cholangioskopie soll wegen des Risikos einer Luftembolie obligat unter CO2-Insufflation oder Wasserspülung durchgeführt werden.

Starker Konsens 


\section{Empfehlung:}

Die gesetzlichen Regelungen des Strahlenschutzes sollen sowohl für die Patienten als auch für das Personal strikt eingehalten werden. Starker Konsens

\section{Empfehlung:}

Die ERCP soll in Sedierung durchgeführt werden.

Starker Konsens

\subsubsection{Durchführung}

\section{Empfehlung:}

Für die Standard-ERCP sollen Duodenoskope mit Seitblickoptik verwendet werden.

Starker Konsens

\subsubsection{Medikamentöse Komplikationsprophylaxe}

\section{Empfehlung:}

Zur medikamentösen Prophylaxe einer Post-ERCP-Pankreatitis (PEP) soll $100 \mathrm{mg}$ Diclofenac oder $100 \mathrm{mg}$ Indomethacin rektal als Suppositorium vor oder unmittelbar nach der ERCP appliziert werden.

\section{Starker Konsens}

\subsubsection{Prozedurabhängige Komplikationsprophylaxe}

\section{Empfehlung:}

Die Kanülierung des Gallen- und Pankreasganges bei nicht papillotomierten Patienten sollte primär mit Führungsdraht erfolgen. Starker Konsens

\section{Empfehlung:}

Die Kontrastmittelfüllung des Pankreasganges sollte bei geplanten Interventionen am Gallengang vermieden werden.

Starker Konsens

\section{Empfehlung:}

Bei erhöhtem Risiko für eine PEP sollte zusätzlich zur NSAR-Prophylaxe die passagere Einlage eines Pankreasgangstents in Erwägung gezogen werden.

Starker Konsens

\section{Empfehlung:}

Ist eine primäre Intubation mit dem Katheter/Draht nicht möglich, sollte je nach Dringlichkeit der Intervention die ERCP-Sitzung beendet und in den Folgetagen wiederholt werden.

\section{Starker Konsens}

\section{Empfehlung:}

Alternativ kann durch einen Vorschnitt („precut“) die Erfolgsrate der Intubation erhöht werden. Dazu können verschiedene technische Varianten angewandt werden.

Starker Konsens

\section{Empfehlung:}

Ein Precut sollte durch einen erfahrenen Untersucher oder unter Anleitung durch einen erfahrenen Untersucher erfolgen.

Starker Konsens

\subsubsection{Alternative Zugänge}

\section{Empfehlung:}

Ist der Zugang zu den Gallenwegen mit dem Duodenoskop auf dem oralen Weg wegen geänderter anatomischer Situationen nach Voroperationen nicht erreichbar, sollte ein Versuch mit einem langen Endoskop oder Ballonenteroskop erfolgen.

Starker Konsens

\section{Empfehlung:}

Bei Versagen des transpapillären Zuganges zu den Gallenwegen kann ein Rendezvous mittels PTC durchgeführt werden (siehe Kapitel 4.12 PTCD).

Starker Konsens

\section{Empfehlung:}

Als weitere Alternative kann der Zugang zu Gallenwegen oder dem Pankreasgang bei gegebener Indikation mittels Rendezvous über einen endosonografischen Zugang erreicht werden (siehe Kapitel: 4.11.3 EUS-gestützte Gallengangs- und Pankreasdrainage).

Starker Konsens

\subsubsection{Papillotomie}

\section{Empfehlung:}

Standard zur Papillotomie sollte der Einsatz eines durch Führungsdraht geführten Papillomiekatheters sein.

Starker Konsens

\section{Empfehlung:}

Bei der Sphinkterotomie sollte ein gemischter Schneidestrom zur Reduktion von Blutungskomplikationen eingesetzt werden.

Starker Konsens

\section{Empfehlung:}

Die Länge der Sphinkterotomie soll sich nach anatomischen Gegebenheiten und der geplanten Intervention richten.

Starker Konsens

\section{Empfehlung:}

Die Papillendilatation mit kleinen Ballons - als Alternative zur Sphinkterotomie - sollte nur in begründeten Einzelfällen durchgeführt werden.

Starker Konsens

\section{Empfehlung:}

Eine Empfehlung für die Auswahl eines bestimmten Führungsdrahtes für Interventionen am pankreatobiliären System kann anhand der derzeitigen Evidenz nicht ausgesprochen werden. Starker Konsens

\subsubsection{Choledocholithiasis}

\section{Empfehlung:}

Die primäre ERCP sollte nur bei Patienten mit gesicherter oder mit hoher Wahrscheinlichkeit für eine Choledocholithiasis erfolgen. Starker Konsens

\section{Empfehlung:}

Die ERCP mit Papillotomie und Steinextraktion soll als Standardverfahren zur Behandlung der Choledocholithiasis eingesetzt werden. Starker Konsens

\section{Empfehlung:}

Bei großen Steindurchmessern sollte die mechanische Lithotripsie als Methode der ersten Wahl verwendet werden. Als Alternative ist die Papillendilatation mit großlumigen Ballons zu erwägen. Starker Konsens 


\section{Empfehlung:}

Ist eine primäre Steinextraktion trotz mechanischer Lithotripsie nicht möglich, sollten andere intra- oder extrakorporale Lithotripsiemethoden eingesetzt werden.

Starker Konsens

\section{Empfehlung:}

Gelingt bei der ERCP nicht die komplette Beseitigung von Steinen oder Fragmenten, sollte eine Endoprothese zur Sicherung des Galleabflusses eingelegt werden. Dies kann bei inoperablen Patienten auch die definitive Therapie sein.

Starker Konsens

\subsubsection{ERCP-gesteuerte pankreatikobiliäre Gewebediagnostik Empfehlung:}

Für die Entnahme von intraduktalen Biopsien aus den Gallenwegen oder dem Pankreasgang in der ERCP können Zangenbiopsien und/oder Bürstenzytologien unter fluoroskopischer Kontrolle entnommen werden. Hier sollte eine ausreichende Anzahl von Biopsien bzw. Bürstenzytologiedurchgängen erfolgen.

Starker Konsens

\subsubsection{Endoskopische pankreatikobiliäre Stenteinlage Empfehlung:}

Zur Ableitung und Therapie von Stenosen des pankreatikobiliären Systems sowie zur Therapie einer Gallengangs- oder Pankreasgangleckage können bei gegebener Indikation Kunststoffendoprothesen und selbst expandierende Metallstents Anwendung finden.

Starker Konsens

\section{Empfehlung:}

Die Einlage einer Kunststoffendoprothese oder eines Metallstents kann auch ohne eine endoskopische Sphinkterotomie erfolgen.

Starker Konsens

\section{Empfehlung:}

Bei Vorliegen einer hochgradigen rigiden Stenose kann vor dem Stenting eine Bougierung oder Ballondilatation auf mindestens den Durchmesser des einzulegenden Kunststoffstents/bzw. Legekatheters bei SEMS erforderlich sein.

Starker Konsens

\section{Empfehlung:}

Die erforderliche Stentlänge soll sich nach der Lage der Stenose bzw. der Länge des zu überbrückenden Segmentes richten und sollte durch Ausmessen bestimmt werden.

Starker Konsens

\section{Empfehlung:}

Ein Wechsel der Kunststoffendoprothese soll programmiert nach 3 Monaten und bei Komplikationen (Cholangitis, fehlender Abfall des Bilirubins) erfolgen. Die Einlage mindestens einer 10 French Kunststoffendoprothese sollte zur Verlängerung der Offenheitsrate angestrebt werden. Eine Begleittherapie mit UDCA und oder Antibiotika zur Prophylaxe einer Stentokklusion soll nicht erfolgen.

Starker Konsens

\section{Empfehlung:}

Die Auswahl des Stents (Kunststoffendoprothese oder SEMS gecovert/teil-/ungecovert) soll sich nach der zugrunde liegenden Indikation, Lokalisation der Stenose richten.

Starker Konsens

\subsection{Biliäre benigne Stenose}

\section{Empfehlung:}

Die endoskopische Therapie von benignen biliären Stenosen sollte aus einem Multistenting mit Kunststoffendoprothesen über 12 Monate bestehen.

Starker Konsens

\section{Empfehlung:}

Alternativ kann bei extrahepatischen benigenen biliären Stenosen die Einlage eines voll gecoverten SEMS erfolgen.

Starker Konsens

\section{Empfehlung:}

Partiell gecoverte oder ungecoverte Metallstents sollen bei benigner Indikation im pankreatobiliären System nicht angewandt werden.

Starker Konsens

\section{Empfehlung:}

Dominante Strikturen (Stenosen der extrahepatischen Gallenwege, Hilus oder zentrale Anteile der Ducti hepatici) bei einer primär sklerosierenden Cholangitis (PSC) sollten eher Ballon dilatiert als endoprothetisch versorgt werden.

Starker Konsens

\subsection{Biliär maligne Stenose \\ Empfehlung:}

Eine präoperative endoskopische Ableitung einer malignen DHCStenose sollte nur bei gegebener Indikation (Hohes Bilirubin, Cholangitis, späterer Op-Zeitpunkt, neoadjuvante Therapie) erfolgen. Hier können Kunststoffendoprothesen oder alternativ ein voll gecoverter Metallstent platziert werden.

\section{Starker Konsens}

\section{Empfehlung:}

Zur palliativen Ableitung einer malignen extrahepatischen biliären Stenose soll primär eine endoskopische Stenteinlage erfolgen. Bei Patienten mit einer Lebenserwartung von über 4 Monaten sollte die Einlage eines SEMS gewählt werden. Hier kann ein voll-, teil oder ungecoverter Metallstent eingesetzt werden.

Starker Konsens

\section{Biliär hiläre Tumore}

\section{Empfehlung:}

Bei biliär hilären Tumoren soll vor einer elektiven ersten biliären endoskopischen Dekompression eine suffiziente Bildgebung zur weiteren Therapieplanung erfolgen. Hier erscheint die MRCP bzgl. der lokalen Tumorausdehnung der CT überlegen.

Starker Konsens

\section{Empfehlung:}

Vor der endoskopischen Ableitung hilärer Tumore sollte die gezielte Drainage der Lebersegmente anhand einer aktuellen Bildgebung geplant werden. Präoperativ soll mindestens der verbleibende Leberlappen suffizient drainiert werden. Palliativ sollen mindestens $50 \%$ des Lebervolumens drainiert werden. Starker Konsens

\section{Empfehlung:}

Zur Drainage hilärer Tumore sollte die Einlage von Kunststoffendoprothesen Standard sein. Die palliative Drainage kann bei einer 
Lebenserwartung von über 4 Monaten in ausgewählten Fällen auch durch nicht gecoverte SEMS erfolgen.

Starker Konsens

\subsection{Lokalablative Therapie von biliären Tumorstenosen}

\section{Empfehlung:}

Zur palliativen endoskopischen Therapie des extrahepatischen Cholangiokarzinoms kann eine fotodynamische Therapie zusätzlich zur Stentversorgung erfolgen. Eine Empfehlung zur Auswahl eines bestimmten Fotosensitizers kann anhand der vorliegenden Daten nicht gegeben werden.

Starker Konsens

\section{Empfehlung:}

Zur Radiofrequenzablation kann aufgrund der aktuellen Datenlage keine abschließende Beurteilung abgegeben werden.

\section{Starker Konsens}

\subsection{Gallengangsleckage}

\section{Empfehlung:}

Zur endoskopischen Therapie von Gallengangsleckagen nach Cholezystektomie, Lebertransplantation oder leberchirurgischen Eingriffen soll primär eine endoskopische Galleableitung erfolgen. In Abhängikeit von Lokalisation und Größe der Leckage sollte eine endoskopischer Sphinkerotomie mit Einlage einer Endoprothese oder die alleinige Sphinkterotomie erfolgen.

Starker Konsens

\subsection{Pankreasgangstenosen}

\section{Empfehlung:}

Zur endoskopischen Therapie von symptomatischen Pankreasgangstenosen im Rahmen einer chronischen Pankreatitis können Dilatation und Stenteinlage durchgeführt werden. Dabei sollte die Dilatation und Stenteinlage nach endoskopischer Sphinkterotomie des Pankreassphinkters erfolgen.

Starker Konsens

\section{Empfehlung:}

Die Einlage einer 10 F Kunststoffendoprothese sollte zu Dilatationszwecken angestrebt werden. Zur weiteren Dilatation rigider Stenosen kann ein Multistenting mit mehreren Kunststoffendoprothesen erfolgen. Eine definitive Empfehlung über die Zeitdauer der endoskopischen Dilatationstherapie kann anhand der bestehenden Evidenz nicht festgelegt werden.

Starker Konsens

\section{Empfehlung:}

Die Einlage eines voll gecoverten SEMS in den Pankreasgang kann in ausgewählten Fällen zur endoskopischen Dilatation von Gangstenosen erfolgen.

Starker Konsens

\section{Empfehlung:}

Zur passageren Schienung einer Pankreasgangruptur nach Trauma oder zur transpapillären Ableitung einer Pseudozyste kann die Einlage einer Kunststoffendoprothese erfolgen.

Starker Konsens

\subsubsection{Cholangioskopie}

\section{Empfehlung:}

Eine Cholangioskopie kann über ein Duodenoskop in „Mutter-Baby-Technik“ oder als direkte perorale Cholangioskopie mit ultradünnen Endoskopen erfolgen. Je nach verwendetem System soll die Cholangioskopie durch ein oder zwei Untersucher durchgeführt werden. Voraussetzung für die Cholangioskopie sollte eine adäquat weite Papillotomie sein. Die Cholangioskopie soll unter Wasserspülung oder CO2-Insufflation durchgeführt werden. Starker Konsens

\subsubsection{Prozedurabhängige Nachsorge \\ Empfehlung:}

Eine stationäre Überwachung sollte in Abhängigkeit von dem individuellen Risikoprofil des Patienten sowie den durchgeführten Interventionen und dem Verlauf der Untersuchung erfolgen.

Starker Konsens

\section{Empfehlung:}

Bei beschwerdefreien Patienten ohne klinischen Anhalt für Komplikationen soll die Routinebestimmung von Pankreasenzymen im Serum nicht erfolgen.

Starker Konsens

\subsubsection{Spezifische Qualitätsindikatoren ERCP (॰ Tab. 15)}

\section{Tab. 15}

\begin{tabular}{l} 
Qualitätsindikatoren \\
\hline präprozedural \\
\hline Frequenz der Antibiotikaprophylaxe bei gegebener Indikation \\
\hline Frequenz der PEP-Prophylaxe bei Risikopatienten/Risikointerventionen \\
intraprozedural \\
\hline Frequenz des Kanülierungserfolges für die Gallengänge bei naiver Papille \\
und normaler Ganganatomie \\
\hline Frequenz einer erfolgreichen Stentplazierung in den DHC bei DHC- \\
Stenose unterhalb der hilären Bifurkation \\
Frequenz der erfolgreichen Steinentfernung bei Gallengangssteinen \\
unter 10 mm und normaler Ganganatomie \\
Frequenz der Erfassung von Durchleuchtungszeit und Dosis \\
postprozedural \\
Frequenz spezifischer Komplikationen: PEP, Blutung nach Papilloto- \\
momie, Perforation
\end{tabular}

\subsection{Diagnostische Endosonografie und endosonogra-} fisch gestützte Feinnadelpunktion

4.9.1 Spezielle Kontraindikationen

\section{Empfehlung:}

Das Vorliegen einer höhergradigen Stenose im Bereich des erforderlichen Passageweges des Echoendoskops ist eine spezielle Kontraindikation. Daher sollte vor einer EUS im oberen GI-Trakt bei Patienten mit Dysphagie oder bekannten Stenosen eine konventionelle ÖGD durchgeführt werden. Hier kann im Einzelfall individuell entschieden werden, ob eine interventionelle Therapie der Stenose zur Ermöglichung der Passage des EUS-Geräts sinnvoll ist.

Starker Konsens

\section{Empfehlung:}

Die endosonografische Feinnadelbiopsie zystischer Mediastinalläsionen sollte vermieden werden.

Starker Konsens 


\subsubsection{Spezielle Vorbereitung}

\section{Empfehlung:}

Die Vorbereitung zur diagnostischen Endosonografie am oberen GI-Trakt soll der Vorbereitung zur Ösophagogastroduodenoskopie (ÖGD) entsprechen, die Vorbereitung zur Endosonografie im Kolon denen zur Koloskopie sowie die Vorbereitung zur rektalen Endosonografie mit starrem Gerät denen zur Rektoskopie. Starker Konsens

\subsubsection{Durchführung}

4.9.3.1 Echoendoskope/Instrumente

\section{Empfehlung:}

Die Verwendung von Radial- und Longitudinalscanner bzw. Minisonde sollte von der jeweiligen Indikation und von der lokalen Expertise und Verfügbarkeit abhängig gemacht werden. Starker Konsens

\subsubsection{Endosonografische Feinnadelpunktion (EUS-FNP)}

\section{Empfehlung:}

Die endosonografische Feinnadelpunktion sollte zur feingeweblichen Diagnose pathologischer Befunde des Verdauungssystems, umgebender Organe und benachbarter Lymphknoten immer dann zum Einsatz kommen, wenn:

- zu erwarten ist, dass das Ergebnis das diagnostische oder therapeutische Management des Patienten beeinflusst,

- die Läsion endosonografisch gestützt mit vertretbarem Risiko erreichbar ist und

- weniger invasive Methode zur Materialgewinnung nicht verfügbar oder ohne Ergebnis geblieben sind.

Starker Konsens

\section{Statement:}

Für die Nadelwahl zur diagnostischen Punktion von Lymphknoten und anderen Läsionen mit Ausnahme solider Pankreasläsionen können keine evidenzbasierten Empfehlungen gegeben werden.

Starker Konsens

\section{Empfehlung:}

Bei der diagnostischen Punktion von soliden Pankreasläsionen sollte die Nadelwahl (vorrangig) von der Zielläsion abhängig gemacht werden.

Starker Konsens

\section{Empfehlung:}

Für solide und zystische Pankreasläsionen sowie für Lymphknoten können 22 G-Nadeln alternativ zu 25 G-Nadeln Verwendung finden. Starker Konsens

\section{Empfehlung:}

Für drahtgestützte therapeutische Interventionen sollen 19 GAspirationsnadeln genutzt werden.

Starker Konsens

\section{Empfehlung:}

Bei Indikation für erweiterte pathologische Untersuchungen sollten EUS-Punktionsnadeln und/oder Punktionstechniken verwendet werden, die die Möglichkeit bieten Gewebezylinder zu gewinnen. Starker Konsens

\section{Empfehlung:}

Die EUS-FNP mit Standardaspirationsnadeln kann auch ohne Verwendung eines Mandrins durchgeführt werden.

Starker Konsens
Empfehlungen:

Die EUS-FNP solider Läsionen kann mit oder ohne Ausübung von Sog durchgeführt werden. Solide Pankreasläsionen sollten im Regelfall mit Sog (Unterdruck mittels Aspirationsspritze oder Slow pull-Technik) aspiriert werden.

Starker Konsens

Bei der EUS-FNP von Lymphknoten und anderen hypervaskulären Läsionen kann die EUS-FNP ohne Sog eine die zytologische Diagnose beeinträchtigende Blutkontamination verhindern.

Starker Konsens

Zysten, Ergüsse und andere liquide Läsionen sollen unter kontinuierlichem Sog aspiriert werden.

Starker Konsens

\section{Empfehlung:}

Das bei der EUS-FNP gewonnene Material kann sowohl mit einem Mandrin als auch durch Aussprühen mit Luft aus der Nadel entfernt werden.

Alternativ kann in Abhängigkeit von der zytopathologischen Weiterverarbeitung mit $\mathrm{NaCl} \mathrm{0,9 \%} \mathrm{oder} \mathrm{Cytolyt} \mathrm{ausgespült} \mathrm{werden.}$

Starker Konsens

\section{Empfehlung:}

In Zentren, in denen die Ausbeute an diagnostisch adäquatem Material bei der EUS-FNP unbefriedigend ist, sollten der gesamte Prozess der EUS-FNP (Materialgewinnung, Materialverarbeitung, zytopathologische Befundung) analysiert und Maßnahmen zur Verbesserung der diagnostischen Effizienz geprüft werden.

Starker Konsens

\subsubsection{Adjuvante Techniken}

\section{Empfehlung:}

Die endosonografische Elastografie kann als komplementäre Methode zur Charakterisierung fokaler Pankreasläsionen eingesetzt werden.

Konsens

\section{Empfehlung:}

In Ergänzung zu den etablierten B-Bild-Kriterien kann die endosonografische Elastografie zur Charakterisierung von Lymphknoten (beispielsweise im Rahmen des Stagings gastrointestinaler Tumoren) eingesetzt werden.

Starker Konsens

\section{Empfehlung:}

Durch Identifikation von durch ihre hohe Gewebehärte für eine maligne Infiltration suspekten Lymphknoten oder Lymphknotenarealen kann die endosonografische Elastografie den zielgerichteten Einsatz der EUS-FNP unterstützen.

Starker Konsens

\section{Empfehlung:}

Die kontrastverstärkte Endosonografie kann als komplementäre Methode zur Charakterisierung solider und zystischer fokaler Pankreasläsionen eingesetzt werden.

Starker Konsens

\subsubsection{Prozedurabhängige Nachsorge Empfehlung:}

Nach rein diagnostischer Endosonografie am oberen bzw. unteren Gastrointestinaltrakt sollen die gleichen Nachsorgekriterien wie für den Einsatz der oberen bzw. unteren diagnostischen En- 
doskopie Anwendung finden. Es sollte, insbesondere nach transduodenaler Endosonografie, auf das Vorliegen klinischer Zeichen für eine Hohlraumperforation geachtet werden.

Starker Konsens

\section{Empfehlung:}

Nach EUS-FNP solider und zystischer Läsionen sollte in Abhängigkeit von dem individuellen Risikoprofil des Patienten, den Charakteristika der Zielläsion sowie dem Verlauf des Eingriffs über eine stationäre Nachsorge entschieden werden. Konsens

\subsubsection{Spezifische Qualitätsindikatoren EUS (o Tab. 16)}

Tab. 16

\begin{tabular}{l} 
Qualitätsindikatoren \\
präprozedural \\
Antibiotikaprophylaxe bei EUS-FNP zystischer Pankreasläsionen \\
intraprozedural \\
Dokumentation von entsprechend der Indikation relevanten anatomischen \\
Strukturen \\
Staging gastrointestinaler Tumoren und von malignen Pankreasneoplasien \\
entsprechend der aktuell gültigen TNM-Klassifikation bzw. bei gastrointes- \\
tinalen Lymphomen der modifizierten Ann Arbor-Klassifikation \\
\hline Dokumentation von Größe und Schichtenzuordnung subepithelialer Tumoren \\
\hline Dokumentation von Größe und Echogenitätscharakteristika von pathologi- \\
schen Lymphknoten und anderen extraintestinalen Raumforderungen \\
\hline Dokumentation der maximalen Pankreasgang- und Gallengangsweite \\
sowie intraduktaler Strukturen bei pankreatobiliären Fragestellungen \\
Einsatz der EUS-FNP im Staging gastrointestinaler Tumoren und des nicht- \\
kleinzelligen Lungenkarzinoms entsprechend der TNM-Klassifikation \\
\hline EUS-FNP: Dokumentation von Nadeltyp, Nadeldurchmesser, Anzahl der \\
Nadelpassagen und Materialverarbeitung \\
\hline postprozedural \\
\hline EUS-FNP solider Läsionen: Häufigkeit der Gewinnung diagnostisch adäqua- \\
ten Materials \\
EUS-FNP solider Läsionen: Häufigkeit diagnostisch konklusiver Befunde \\
(Kategorien: maligne, benigne, neoplastisch) bei Gewinnung adäquaten \\
Materials \\
\hline Häufigkeit prozeduraler Komplikationen nach EUS, EUS-FNP und EUS-ge- \\
stützten therapeutischen Interventionen
\end{tabular}

\subsection{EUS-gestützte Drainage pankreatitisassoziierter} Flüssigkeitsansammlungen

\subsubsection{Spezielle Vorbereitung}

\section{Empfehlung:}

Vor jeder endoskopischen Drainage einer zystischen Pankreasläsion sollte eine sorgfältige Anamnese hinsichtlich der aktuellen klinischen Beschwerden und der Vorerkrankungen erhoben werden und eine suffiziente Bildgebung des Abdomens für Differenzialdiagnose, Therapieplanung und als Ausgangsbefund des therapeutischen Follow-up erfolgen.

Starker Konsens

\subsubsection{Durchführung}

4.10.2.1 Technik des endoskopischen Vorgehens

\section{Empfehlung:}

Bei der endoskopischen Drainage von Pseudozysten und anderen pankreatitisassoziierten Flüssigkeitsansammlungen sollte die Technik des endoskopischen Vorgehens von der Lokalisation der Pseudozyste/Flüssigkeitsansammlung, der Ganganatomie, dem
Nachweis einer Gangassoziation sowie weiteren patientenbezogenen Kriterien (Infektion, portale Hypertension, Pelottierungseffekt der Pseudozyste im oberen Verdauungstrakt) abhängig gemacht werden.

Konsens

\section{Empfehlung:}

Eine transpapilläre Drainage kann erfolgen, wenn die Pseudozyste oder Flüssigkeitsansammlung mit dem Pankreasgang kommuniziert. Dabei sollte nach Sondierung des Pankreasganges eine pankreatische Sphinkerotomie, die Dilatation von der Pseudozyste/ Flüssigkeitsansammlung nachgeschalteten Pankreasgangstrikturen und die Einlage einer Kunststoffendoprothese erfolgen. Bei größeren Pseudozysten oder Flüssigkeitsansammlungen, die über den Pankreasgang zu sondieren sind, kann die Einlage eines Kunststoffpigtails bis in die Pseudozyste versucht werden.

Starker Konsens

\section{Empfehlung:}

Die transmurale Drainage von Pseudozysten/pankreatitisassoziierten Flüssigkeitsansammlungen sollte EUS-gestützt erfolgen (DGVS GL CP). Bei deutlicher Impression der Wandung des Gastrointestinaltrakts, Fehlen einer portalen Hypertension und geringem Blutungsrisiko kann die Drainage auch nach vorheriger diagnostischer Endosonografie konventionell-endoskopisch durchgeführt werden.

\section{Starker Konsens}

\section{Empfehlung:}

Die endosonografische Drainage von Pseudozysten/pankreatitisassoziierten Flüssigkeitsansammlungen sollten abhängig von deren Lokalisation bevorzugt transgastral oder transduodenal erfolgen. Konsens

\section{Empfehlung:}

Die transmurale EUS-gestützte Drainage von Pseudozysten und pankreatitisassoziierten Flüssigkeitsansammlungen kann in verschiedenen Techniken (Punktion-Dilatations-Technik, Diathermie) durchgeführt werden.

\section{Starker Konsens}

\section{Empfehlung:}

Für die transmurale Drainage der pankreatitisassoziierten Flüssigkeitsansammlung können Kunstoffendoprothesen oder voll gecoverte selbstexpandierende Metallstents verwendet werden. Starker Konsens

\section{Empfehlung:}

Die Liegedauer von Kunststoffstents soll mindestens 6 Wochen betragen, um eine gute Langzeitregression von Pankreaspseudozysten und anderen pankreatischen Flüssigkeitskollektionen zu gewährleisten. Die Einlage von mehreren Stents kann die Langzeitregressionsrate ebenfalls positiv beeinflussen.

\section{Starker Konsens}

\subsubsection{Endoskopische Therapie von infizierten Pankreasnekrosen} Empfehlung:

Die endoskopische Drainage von peripankreatischen und Pankreasparenchymnekrosen soll bei Nachweis oder dringendem Verdacht einer Infektion möglichst nach Demarkation der Nekrose und Kapselbildung erfolgen, dies bedingt einen Zeitabstand von mindestens 4 Wochen zum Beginn der akuten Pankreatitis. 
Eine Drainage infizierter nekrotischer Flüssigkeitskollektionen zu einem früheren Zeitpunkt kann bei instabilen Patienten charakterisiert durch SIRS und Multiorganversagen mit klinischer Verschlechterung trotz maximaler intensivmedizinischer Therapie erwogen werden.

Starker Konsens

\section{Empfehlungen:}

Bei gegebener Indikation für eine endoskopische Therapie von demarkierten Pankreasnekrosen (WON) soll in einem minimal invasiven „Step up“-Protokoll entweder durch transmurale endoskopische Drainage ggf. gefolgt von einer direkten endoskopischer Nekrosektomie interveniert werden oder durch perkutane Katheterdrainage ggf. gefolgt von einem minimal invasiven videoendoskopisch assistierten retroperitonealen Debridement. Wird die Nekrose endoskopisch nur partiell erreicht, kann eine Kombination aus endoskopischer und perkutaner Drainage/Nekrosektomie erfolgen.

Starker Konsens

\section{Empfehlung:}

Bei endoskopischer Therapie von demarkierten Pankreasnekrosen (WON) mit und ohne Infektion soll die Technik der endoskopisch-transmuralen Drainage deren Inhalt und Größe Rechnung tragen. Sie kann mit einer Spülbehandlung über einen perkutanen Zugang oder eine nasozystische Sonde bzw. mit einer direkten endoskopischen Nekrosektomie kombiniert werden.

Starker Konsens

\section{Empfehlung:}

Die endoskopische Therapie von demarkierten Pankreasnekrosen (WON) sollte in Kliniken mit entsprechender lokaler Expertise für endoskopische, EUS-gestützte und perkutane Interventionen durchgeführt werden (Endoskopie, interventionelle Radiologie, Chirurgie).

Konsens

4.10.2.3 EUS-gestützte Drainage von nicht mit einer Pankreatitis assoziierten Flüssigkeitsansammlungen

\section{Empfehlung:}

Die EUS-gestützte Drainage von nicht mit einer akuten oder chronischen Pankreatitis assoziierten Flüssigkeitsansammlungen (postoperative Verhalte, Abszesse) kann alternativ zu perkutanen minimal invasiven und operativen Verfahren eingesetzt werden. Starker Konsens

\subsection{Addendum: EUS-gestützte therapeutische Interventionen \\ 4.11.1 EUS-gestützte prätherapeutische Tumormarkie- rung \\ Empfehlung:}

Die EUS-gestützte Platzierung von Edelmetallmarkern kann zur Markierung von Tumoren und Lymphknoten vor operativer oder stereotaktischer Radiotherapie eingesetzt werden.

Starker Konsens

\subsubsection{EUS-gestützte Blockade/Neurolyse des Plexus coeliacus \\ Empfehlung:}

Bei Patienten mit Schmerzsyndrom bei irresektablem Pankreaskarzinom und anderen malignen Erkrankungen des oberen Bauch- raums sollte die Einbeziehung der endosonografisch gestützten Neurolyse des Plexus coeliacus (EUS-CPN) bzw. des Ganglion coeliacus in die multimodale Schmerztherapie erwogen werden.

Starker Konsens

\section{Empfehlung:}

Bei Patienten mit chronischem Schmerzsyndrom im Rahmen einer chronischen Pankreatitis kann die Einbeziehung der endosonografisch gestützten Blockade des Plexus coeliacus (EUS-CPB) in die multimodale Schmerztherapie erwogen werden, wenn eine operative Therapie nicht gewünscht wird oder aus anderen Gründen nicht infrage kommt.

Starker Konsens

\section{Empfehlung:}

Bei endosonografischer Darstellbarkeit sollten endosonografisch gestützte Plexusneurolyse oder -blockade als direkte Injektionstherapie der Ganglien durchgeführt werden.

Starker Konsens

Alternativ und insbesondere bei fehlender endosonografischer Darstellbarkeit der Ganglien können endosonografisch gestützte Plexusneurolyse oder -blockade sowohl als bilaterale oder als zentrale Injektion an der Basis des Truncus coeliacus oder unter Einbeziehung des Plexus mesentericus superior (broad plexus neurolysis) erfolgen.

Starker Konsens

\subsubsection{EUS-gestützte Gallengangs- und Pankreasgang- drainage \\ EUS-gestützte Gallengangsdrainage}

\section{Empfehlung:}

Die EUS-gestützte Gallengangsdrainage (EUS-CD) als Rendezvousverfahren oder mit transmuraler Stenteinlage kann abhängig von der konkreten klinischen und anatomischen Situation sowie der lokalen Expertise und unter Abwägung der möglichen Alternativverfahren (PTCD, operative Verfahren) in ausgewählten klinischen Einzelfällen in Erwägung gezogen werden.

Starker Konsens

\section{EUS-gestützte Pankreasgangdrainage}

\section{Empfehlung:}

Die endosonografisch gestützte Pankreasgangdrainage (EUS-PD) kann abhängig von der konkreten klinischen und anatomischen Situation sowie der lokalen Expertise und unter Abwägung der möglichen Alternativverfahren (chirurgische Drainageverfahren) in ausgewählten klinischen Einzelfällen in Erwägung gezogen werden.

Starker Konsens

\subsubsection{EUS-gestützte Tumortherapie Empfehlungen:}

EUS-gestützte Verfahren zur Tumorablation und Brachytherapie sollen nur innerhalb von Studien angewandt werden.

Starker Konsens

\section{Empfehlung:}

EUS-gestützte Verfahren zur Injektion antineoplastischer Substanzen sollen außerhalb von Studien nicht eingesetzt werden. Starker Konsens 


\section{Empfehlung:}

Die EUS-gestützte Ablationstherapie zystischer Pankreasneoplasien soll außerhalb von Studien nicht durchgeführt werden. Starker Konsens

\subsection{Perkutane transhepatische Cholangiografie (PTC)} und Cholangiodrainage (PTCD)

4.12.1 Spezielle Kontraindikationen

\section{Empfehlung:}

Relative Kontraindikationen für die Anlage einer PTCD können sein:

- schwere nicht korrigierbare Gerinnungsstörungen (Quick $<50 \%$, bzw. INR $>1,6$; Thrombozyten $<50000 / \mu \mathrm{l}$ )

- Schwangerschaft

- ausgeprägter, nicht ausreichend punktierbarer Aszites

- unsicherer Drainageweg

- multifokale intrahepatische Segmentstenosen

Starker Konsens

\subsubsection{Spezielle Vorbereitung}

\section{Empfehlung:}

Vor der PTC(D) soll eine Bildgebung (Sonografie; MRT mit MRCP, $\mathrm{CT}$ ) zur Darstellung der intrahepatischen Gallenwege erfolgen. Starker Konsens

\section{Empfehlung:}

Die gesetzlichen Regelungen des Strahlenschutzes sollen sowohl für die Patienten als auch für das Personal eingehalten werden. Starker Konsens

\subsubsection{Durchführung Empfehlung:}

Die PTC/PTCD sollte in Analgesie, Analgosedierung oder Intubationsnarkose durchgeführt werden.

Starker Konsens

\section{Empfehlung:}

Die PTC/PTCD sollte in Rückenlage durchgeführt werden. Alternativ kann die PTCD-Anlage bei geplantem Rendezvousmanöver und Zugang von rechts auch primär in Bauchlage erfolgen.

Starker Konsens

\section{Empfehlung:}

Die PTCD und PTC soll unter sterilen Bedingungen erfolgen. Dies soll das sterile Arbeiten des Untersuchers und das sterile Instrumentieren der Pflegeassistenz sowie eine nicht sterile Pflegeassistenz als Springer umfassen.

Starker Konsens

\section{Empfehlung:}

Um einen sicheren Zugangsweg zu gewährleisten, sollte unter sonografischer oder radiografischer Kontrolle punktiert werden. Starker Konsens

\section{Empfehlung:}

Zur perkutanen Cholangiografie sollte eine dünnlumige (21 - 22 G) Hohlnadel verwendet werden. Die erfolgreiche Initialpunktion kann durch Rückfluss von Galle aus der Nadel oder durch Injektion von Kontrastmittel unter Röntgendurchleuchtung während des Nadelrückzuges erfasst werden.

Starker Konsens

\section{Empfehlung:}

Die Anlage einer PTC-Drainage sollte nach erfolgreicher Punktion des geeigneten Gallenganges in Seldinger-Technik erfolgen. Eine Empfehlung zur Verwendung von Dilatatoren oder Führungsdrähten bestimmter Hersteller kann nicht ausgesprochen werden.

Starker Konsens

\section{Empfehlung:}

Die Anlage einer intern-externen Drainage in den Dünndarm sollte angestrebt werden. Ist dies nicht möglich, sollte zur Dekompression zunächst eine externe Ableitung erfolgen. Art und Größe der initial gewählten Drainage sollen angepasst an das Ausmaß der Gallengangdilatation, an die Beschaffenheit der Stenose und das Risikoprofil des Patienten gewählt werden.

Starker Konsens

\section{Empfehlung:}

Bei bestehender oder vermuteter Cholangitis sollten während der initialen Cholangiografie Galleproben zur mikrobiologischen Kultivierung entnommen werden.

\section{Starker Konsens}

\section{Empfehlung:}

Die erste Traktdilatation zu Erreichung einer ausreichenden PTCD-Größe zu diagnostischen oder therapeutischen Zwecken sollte erst 2 - 3 Tage nach der PTCD-Erstanlage erfolgen. Die gewünschte Zielgröße kann in einer Dilatationssitzung oder in mehreren aufeinanderfolgenden Sitzungen erreicht werden. Dies ist abhängig von der Anatomie der Gallenwege, der gewünschten Zielgröße und dem Risikoprofil des Patienten. Starker Konsens

\section{Empfehlung:}

Eine diagnostische oder therapeutische Cholangioskopie sollte frühestens 5 Tage nach der Traktdilatation erfolgen, um eine ausreichende Traktreifung zu gewährleisten.

Starker Konsens

\section{Statement:}

Therapeutische Interventionen über den perkutanen Zugang umfassen die Behandlung von benignen und malignen Stenosen unter Verwendung von Kunststoffdrainagen, Ballondilatation und selbstexpandierenden Metallstents sowie die perkutane Cholangioskopie mit Gallensteintherapie und lokaler Tumortherapie. Hier kommen die im Kapitel ERCP (Kap. 4.8) beschriebenen Prinzipien zur Anwendung.

Starker Konsens

\subsubsection{Prozedurabhängige Nachsorge Empfehlung:}

Alle Patienten mit PTCD-Neuanlage sollen stationär nachbeobachtet werden.

Starker Konsens

\section{Empfehlung:}

Nach PTCD-Anlage soll eine ausreichende Analgetikagabe erfolgen. Starker Konsens

\section{Empfehlung:}

Ein regelmäßiger (z. B. 2-mal wöchentlicher) Verbandswechsel zur frühzeitigen Erkennung und Vermeidung lokaler Infektionen sowie regelmäßiges Spülen der Prothese sollte gewährleistet werden. 
Bei der Dauertherapie sollte ein regelmäßiger Wechsel der perkutanen Drainagen vorgenommen werden.

Starker Konsens

\subsubsection{Spezifische Qualitätsindikatoren PTCD \\ (o Tab.17)}

\section{Tab. 17}

\begin{tabular}{l} 
Vorschläge für Qualitätsindikatoren PTCD \\
\hline präprozedural \\
\hline Durchführung einer Bildgebung der Gallenwege vor PTC/PTCD \\
\hline intraprozedural \\
\hline PTC-Erfolgsrate für: \\
\hline - Darstellung dilatierter Gallenwege \\
\hline - Darstellung nicht dilatierter Gallenwege \\
\hline PTCD-Erfolgsrate für: \\
\hline - Kanülierung dilatierter Gallenwege \\
\hline - Darstellung dilatierter Gallenwege \\
\hline - Internalisierung bei erfolgreicher Kanülierung \\
\hline
\end{tabular}

\subsection{Flexible Dünndarmendoskopie}

\subsubsection{Spezielle Kontraindikationen}

\subsubsection{Spezielle Vorbereitung}

Empfehlung:

Bei oralem Zugangsweg sollten die Patienten etwa 10 - 12 Stunden nüchtern sein. Die Gabe von Abführlösungen kann erfolgen. Starker Konsens

\section{Empfehlung:}

Bei analem Zugangsweg soll eine besonders effektive Darmlavage erfolgen. Dabei soll die Vorbereitung prinzipiell wie vor Koloskopie erfolgen.

Starker Konsens

\subsubsection{Durchführung \\ Sedierung \\ Empfehlung:}

Die Enteroskopie soll in Sedierung, in Einzelfällen auch in Intubationsnarkose, erfolgen.

Starker Konsens

Röntgendurchleuchtung

\section{Empfehlung:}

Die Verwendung einer radiologischen Kontrolle kann bei schwierigen anatomischen Verhältnissen, klinischen Zeichen der Obstruktion oder dem analen Zugang empfehlenswert sein.

Starker Konsens

CO2-Insufflation

\section{Empfehlung:}

Die Ballonenteroskopie soll unter Verwendung von CO2 erfolgen. Starker Konsens

Messung der Eindringtiefe

Empfehlung:

Die Bestimmung der Eindringtiefe sollte für die ballonassistierten Verfahren auf dem Hinweg abgeschätzt werden.

Starker Konsens
Therapeutische Enteroskopie

\section{Empfehlung:}

Therapeutische Interventionen sollten in der Regel während des Rückzugs erfolgen.

Starker Konsens

\section{Empfehlung:}

Therapeutische Interventionen sollten von einem erfahrenen Endoskopiker mit einer erfahrenen Endoskopieassistenz bzw. unter Supervision eines erfahrenen Enteroskopikers durchgeführt werden. Starker Konsens

\subsubsection{Prozedurabhängige Nachsorge Empfehlung:}

Nach therapeutischer Enteroskopie sollte eine stationäre Überwachung abhängig von der Intervention und dem individuellen Risikoprofil des Patienten erfolgen.

Starker Konsens

\subsection{Kapselendoskopie}

\subsubsection{Spezielle Kontraindikationen}

Schrittmacher/Implantierte Kardioverter (ICDs)

Empfehlung:

Patienten mit Schrittmachern/ICDs sollte bei gegebener Indikation eine Kapselendoskopie nicht vorenthalten werden. Eine explizite Aufklärung soll erfolgen. Eine regelmäßige Neubewertung unter Einbeziehung von Daten zum aktuell eingesetzten Kapselsystem ist erforderlich.

Starker Konsens

Stenosen

\section{Empfehlung:}

Bei klinischem Verdacht auf eine intestinale Stenose soll die Kapselendoskopie erst nach Beweis der Durchgängigkeit mittels vorheriger Patency-Kapseluntersuchung erfolgen.

Lediglich bei unergiebiger Vordiagnostik, bestehendem Operationswunsch, OP-Indikation und OP-Fähigkeit des Patienten im Falle einer Retention kann nach eingehender Aufklärung eine Kapselendoskopie auch bei Stenoseverdacht erfolgen.

Konsens

\section{Schluckstörungen}

\section{Empfehlung:}

Bei Schluckstörungen sollte die endoskopische Einbringung der Kapsel in den Magen oder das Duodenum erfolgen.

Starker Konsens

\subsubsection{Spezielle Vorbereitung \\ Darmreinigung, Entschäumer \\ Empfehlung:}

Eine Darmreinigung sollte vor einer Kapselendoskopie erfolgen, ebenso sollte Simethicon zur besseren Beurteilbarkeit der Dünndarmmukosa verabreicht werden

Starker Konsens

Prokinetika

Empfehlung:

Der generelle Einsatz von Prokinetika vor Kapselendoskopie kann nicht empfohlen werden. Eine gezielte Gabe kann aber sinnvoll sein bei bekannter oder bei mittels Real-time-Viewer beobachteter Magenentleerungsstörung.

Starker Konsens 


\subsubsection{Durchführung}

Kapselsysteme

\section{Statement:}

Anhand der vorliegenden Daten kann keine Empfehlung für oder gegen ein System ausgesprochen werden.

Starker Konsens

\section{Auswertung \\ Automatisierte Abspielgeschwindigkeit und Bildselektion \\ Empfehlung:}

Die Verwendung von Softwaremodi mit Anpassung der Abspielgeschwindigkeit an die Kapselbewegung und mit Unterdrückung redundanter Bilder kann die Auswertung einer Kapselendoskopie beschleunigen. Eine Betrachtung von softwareselektionierten Bildern kann die Diagnosestellung beschleunigen, soll aber die komplette Durchsicht des Videos nicht ersetzen.

Starker Konsens

\section{Farbselektion}

\section{Empfehlung:}

Die Verwendung von Farbselektion kann im Einzelfall bei der Beurteilung von Läsionen hilfreich sein. Sie sollte derzeit aber nicht zum generellen Einsatz bei der Detektion empfohlen werden. Starker Konsens

\subsubsection{Prozedurabhängige Nachsorge Empfehlung:}

Kapseltypen mit Bildübertragung während der Aufzeichnung müssen nicht geborgen werden. Auf die Ausscheidung der Kapsel soll durch die Patienten geachtet werden. Ist das Kolon abgebildet, soll eine routinemäßige Röntgenaufnahme auch bei fehlender Beobachtung der Kapselausscheidung nicht erfolgen.

\section{Starker Konsens}

\section{Empfehlung:}

Ist das Kolon nicht abgebildet, soll der Patient nach der Kapselausscheidung gefragt werden. Wurde diese nicht beobachtet, soll der Ausschluss einer Retention erfolgen.

\section{Starker Konsens}

\section{Empfehlung:}

Eine Kernspintomografie soll bei Patienten mit inkorporierter Videokapsel nicht erfolgen.

Starker Konsens

\subsubsection{Spezifische Qualitätsindikatoren Kapselendoskopie (o Tab. 18)}

\section{Tab. 18}

Qualitätsindikatoren
präprozedural
Vermeidung von Kapselretention
intraprozedural
Beurteilbarkeit der Dünndarmmukosa adäquat
komplette Dünndarmkapselendoskopie (Erreichen des Zökums im
Untersuchungszeitraum)

\subsection{Diagnostische Laparoskopie \\ 4.15.1 Spezielle Kontraindikationen \\ Empfehlung:}

Relative Kontraindikation für die diagnostische Laparoskopie können sein:

- ausgedehnte Verwachsungen durch Voroperationen

- ein erhöhtes Blutungsrisiko bei fortgeschrittener Leberzirrhose Child-Pugh C, ausgeprägter portaler Hypertension oder eingeschränkter plasmatischer Gerinnung und/oder Thrombozytopenie

Starker Konsens

\subsubsection{Spezielle Vorbereitung}

\section{Empfehlung:}

Zur Durchführung einer diagnostischen Laparoskopie mit Leberbiopsie kann bei erniedrigten Thrombozytenzahlen $<50000 / \mu l$ bzw. einem INR > 1,6 eine Substitution erfolgen.

Starker Konsens

\subsubsection{Durchführung \\ Empfehlung:}

Die diagnostische Laparoskopie soll unter sterilen Bedingungen durchgeführt werden. Dies beinhaltet einen sterilen Untersucher und Assistenten, eine sterile Pflegeassistenz sowie eine nicht sterile Pflegeassistenz als Springer.

Starker Konsens

\section{Empfehlung:}

Zur Anlage des Pneumoperitoneums sollte bei der diagnostischen Laparoskopie unter Analgosedierung Lachgas verwendet werden.

Starker Konsens

\section{Empfehlung:}

Zur Anlage des Pneumoperitoneums sollte die Veressnadel in der Regel am Kalk-Punkt eingebracht werden (Minilaparoskopie), alternativ am Monroe-Punkt. Bei Organomegalie oder Verwachsungen bei Voroperationen kann der Zugangsweg nach sonografischer Orientierung variiert werden. Zusätzliche Trokare werden im Einzelfall orientiert eingebracht.

Starker Konsens

\section{Empfehlung:}

Die diagnostische Laparoskopie sollte mit dünnkalibrigen Laparoskopen $(1,9-3,5 \mathrm{~mm})$ durchgeführt werden. Kaliberstärkere Laparoskope können eingesetzt werden.

Starker Konsens

\section{Empfehlung:}

Bei hepatologischen Fragestellungen soll die Leberoberfläche nach makroskopischen Gesichtspunkten beurteilt werden. Zur Punktion soll ein makroskopisch auffälliges Leberareal ausgesucht werden. Eine beidseitige Leberpunktion kann erfolgen.

Starker Konsens

\section{Empfehlung:}

Zur laparoskopischen Organbiopsie können Nadeln nach dem Vakuum- und Schneidbiopsieprinzip verwendet werden. Bei Vorliegen einer Leberzirrhose kann die Verwendung einer Nadel nach dem Schneidebiopsieprinzip vorteilhaft für die diagnostische Ausbeute sein.

Starker Konsens 


\section{Empfehlung:}

Tritt nach laparoskopischer Organpunktion eine starke oder lang anhaltende Blutung aus der Einstichstelle auf, sollen laparoskopische Blutstillungsverfahren (Kompression, Koagulationsverfahren, Fibrinkleber) zur Anwendung kommen.

Starker Konsens

\section{Empfehlung:}

Auffällige Läsionen des Peritoneums sollen biopsiert werden. Starker Konsens

\section{Empfehlung:}

Der Unterbauch sollte bei der diagnostischen Laparoskopie mitbeurteilt werden.

Starker Konsens

\subsubsection{Prozedurabhängige Nachsorge Empfehlung:}

Eine stationäre Überwachung kann in Abhängigkeit von dem individuellen Risikoprofil des Patienten sowie dem Verlauf der Untersuchung erfolgen.

Starker Konsens

\subsubsection{Komplikationen der diagnostischen Laparoskopie}

\subsection{Endoskopie in der Schwangerschaft Empfehlung:}

Vor jeder elektiven Endoskopie in Sedierung oder geplanten Interventionen in der Schwangerschaft soll ein Geburtshelfer konsultiert werden.

Starker Konsens

\section{Empfehlung:}

Zur Durchführung einer Endoskopie in der Schwangerschaft soll die Indikation eng gestellt werden.

Starker Konsens

\section{Empfehlung:}

Die Kapselendoskopie soll bei Schwangeren nicht erfolgen. Konsens

\section{Empfehlung:}

Die Endoskopie bei schwangeren Patientinnen im 2 und 3. Trimenon soll in Linksseitenlage erfolgen.

Starker Konsens

\section{Empfehlung:}

Die Endoskopie bei Schwangeren sollte - falls möglich - im 2. Schwangerschaftstrimenon erfolgen.

Starker Konsens

\section{Empfehlung:}

Bei der Stromapplikation soll die Neutralelektrode so angebracht werden, dass der Uterus sich nicht zwischen dem elektrischen Instrumentarium und der Neutralelektrode befindet. Bipolarer Strom sollte verwendet werden, um eine Stromapplikation auf den Fetus zu vermeiden.

Starker Konsens

\section{Empfehlung:}

Die Strahlenbelastung bei endoskopischen Eingriffen (ERCP/PTCD) soll möglichst gering gehalten werden.

Starker Konsens

\section{Kap. 5 Prozessqualität - Dokumentation von Befunden} $\nabla$

\subsection{Befunddokumentation Empfehlung:}

Bereits vorhandene Daten aus früheren Untersuchungen sollen bei Verlaufskontrollen oder im Rahmen von Nachsorgeuntersuchungen oder auch zur Überprüfung der erbrachten Leistung jederzeit exakt erkennbar sein.

Starker Konsens

\section{Empfehlung:}

Die Dokumentation des gesamten Behandlungsablaufes soll folgende Punkte beinhalten:

1. Patientenidentifikation

2. Untersuchungsindikation

3. Identifikation von Untersucher und Assistenzpersonal

4. Gerätetyp und Geräteidentifikation (Seriennummer)

5. Medikation unter Bezug auf die Dokumentationserfordernisse nach S3-Sedierungsleitlinie

6. Zeiterfassung des Untersuchungs-und Eingriffsprozesses

7. Vollständigkeit und Beurteilbarkeit der Untersuchung a) Vollständigkeit der endoskopischen Untersuchung I. Angabe der Ausdehnung

II. Bei unvollständiger Endoskopie: Angabe der Gründe b) Beurteilbarkeit

8. Endoskopischer Befund nach Standardklassifikationssystem (Normalbefund, Pathologischer Befund)

a) Läsion und ihre Attribute

b) Lokalisation und Ausdehnung (ggf. in Maßeinheiten)

9. Endoskopische Diagnose und Therapie

a) Beschreibung der diagnostischen/therapeutischen Maßnahmen

b) Dokumentation der der entnommenen histologisch aufzuarbeitenden Proben/Gewebe nach Lokalisation

c) Intraprozedurale Komplikationen

d) Empfehlungen zur Nachsorge und zum weiteren Procedere

10. Kodierung von ICD und OPS mittels hinterlegter Vorschlagsdiagnosen mit ICD- und OPS-Schlüssel. Mehrfachnennungen sollen möglich sein.

11. Erfassung der verwendeten Instrumente und Materialien

12. Relevante Bilddateien sollen dem Befundbericht beigefügt sein

Konsens

\subsection{Bild-/Filmdokumentation Empfehlung:}

Es soll eine standardisierte patientenbezogene Bilddokumentation erfolgen. Die Dokumentation und Archivierung von Bild- und Filmmaterial sollte EDV gestützt erfolgen. Konsens

\section{Kap. 6 Pozessqualität - Hygiene und Aufbereitung \\ Empfehlung:}

Unabhängig vom Durchführungsort der Endoskopie sollen die gleichen räumlich-apparativen und personellen Voraussetzungen für die Hygiene erfüllt und die gleichen Hygienemaßnahmen durchgeführt werden.

Starker Konsens 


\subsection{Strukturqualität für Hygiene in der Endoskopie \\ 6.1.1 Sicherheit der Patienten \\ Empfehlung:}

Jeder Patient, der sich endoskopischen Eingriffen unterzieht, hat das Recht, im Rahmen der Behandlung vor Infektionen geschützt zu werden. Da der bakterielle und virale Trägerstatus von Patienten in der Endoskopie oftmals unbekannt ist, soll jeder Patient als potenziell infektiös bzw. als potenzieller Träger angesehen werden.

Starker Konsens

\subsubsection{Sicherheit des Personals Empfehlung:}

Endoskopiefachpersonal und Endoskopiker sollen vor direktem Kontakt mit Körperflüssigkeiten, potenziell infektiösem Material und kontaminierten Instrumenten sowie vor direktem Kontakt mit Prozesschemikalien geschützt werden, um möglichen Infektionen und Gesundheitsproblemen vorzubeugen bzw. zu vermeiden.

Starker Konsens

\subsubsection{Ausbildung und Kompetenzen von Personal Empfehlung:}

Die Mitarbeiter in der Endoskopie sollten regelmäßig Schulungen zur Hygiene und Infektionsprävention erhalten.

Starker Konsens

\section{Empfehlung:}

Der Arbeitgeber soll nur Personal mit der Aufbereitung von Endoskopen und endoskopischem Instrumentarium beauftragen, das über die dafür notwendige Ausbildung, Sachkenntnis und Erfahrung verfügt.

Starker Konsens

\subsubsection{Anzahl des Personals Empfehlung:}

Eine ausreichende Anzahl von qualifiziertem Personal soll zur Verfügung stehen, damit die Empfehlungen zur Hygiene konsequent und korrekt eingehalten werden können.

Starker Konsens

\subsubsection{Räumlich-apparative Voraussetzungen Empfehlung:}

Endoskopieräume sollen über ausreichend Platz und Ablageflächen verfügen, um ein keimreduziertes und ggf. steriles Arbeiten bei Untersuchungen und Eingriffen zu ermöglichen. Ein hygienischer Händewaschplatz und persönliche Schutzausrüstung sollen in jedem Untersuchungsraum vorhanden sein. Flächen sollen leicht zu reinigen und zu desinfizieren sein. Die speziellen Anforderungen an Untersuchungs- und Eingriffsräume sollen erfüllt werden.

Starker Konsens

\section{Empfehlung:}

Die Aufbereitung von flexiblen Endoskopen und Zusatzinstrumentarium soll in separaten, zweckmäßig ausgestatteten Aufbereitungsräumen erfolgen, die eine strikte arbeitstechnische bzw. räumliche Trennung von reinen und unreinen Arbeitsbereichen und Lagerung ermöglichen sowie über eine entsprechende technische Ausstattung und Schutzmaßnahmen verfügen, um eine sichere Aufbereitung nach standardisierten und validierten
Aufbereitungsverfahren zu ermöglichen sowie Infektionen und Kreuzkontaminationen zu vermeiden. Starker Konsens

\subsection{Prozessqualität zur Hygiene in der Endoskopie 6.2.1 Hygienemaßnahmen während Untersuchungen und Eingriffen \\ Empfehlung:}

Bei endoskopischen Eingriffen sollen Endoskopiker und Assistenz zum Schutz vor Kontaminationen flüssigkeitsabweisende Schutzkittel und Schutzhandschuhe tragen.

Wenn mit dem Verspritzen von Körperflüssigkeiten und Blut zu rechnen ist, sollen zusätzlich Augen- und Gesichtsschutz getragen werden.

Bei endoskopischen Eingriffen und perkutanen Eingriffen soll je nach Keimspektrum und Zugangsweg ein keimreduziertes bzw. steriles Arbeiten erfolgen.

Bei Eingriffen unter sterilen Kautelen soll entsprechend sterile Schutzausrüstung getragen werden.

Starker Konsens

\section{Empfehlung:}

Die hygienische Händedesinfektion soll vor Patientenkontakt, vor sauberen/aseptischen Maßnahmen, vor endoskopischen Eingriffen und Punktionen sowie nach Patientenkontakt, Kontakt mit Körperflüssigkeiten und kontaminierten Instrumenten, infektiösem Material und nach Kontakt mit der direkten Patientenumgebung erfolgen.

Starker Konsens

\section{Empfehlung:}

Im Rahmen der Sedierung sollen Medikamente und Infusionslösungen unter aseptischen Bedingungen vorbereitet und zeitnah appliziert werden. Angebrochene Applikationssysteme sollen verworfen werden. Alle Abfüllungen sollen nur zur einmaligen Anwendung bei einem Patienten verwendet werden.

Starker Konsens

\subsubsection{Hygienemaßnahmen während der Aufbereitung von} Medizinprodukten in der Endoskopie

\section{Empfehlung:}

Bei der Aufbereitung von Endoskopen und Zusatzinstrumentarium sollen Mitarbeiter persönliche Schutzausrüstung tragen, bestehend aus:

- langen Schutzhandschuhen, die Schutz vor bakteriologische Kontaminationen und chemische Gefahren bieten

- feuchtigkeitsdichtem, langärmeligem Schutzkittel

- Gesichtsschutz (Mund-Nasen-Schutz, Schutzbrille oder Visier) Wenn an Aufbereitungsbecken ein wirksamer Spritzschutz vorhanden ist, kann auf den Gesichtsschutz verzichtet werden.

\section{Starker Konsens}

\subsubsection{Standardisierte Aufbereitung von flexiblen Endoskopen Empfehlung:}

Endoskope sollen direkt nach Beendigung des Eingriffs vorgereinigt werden, um grobe Verschmutzungen zu entfernen und die korrekte Funktion des Gerätes zu überprüfen.

Der Transport von Endoskopen und Zusatzinstrumentarium soll im geschlossen System erfolgen, um Umgebungskontaminationen zu vermeiden.

Starker Konsens 


\section{Empfehlung:}

Die manuelle Reinigung der Endoskope soll nach standardisierten Protokollen erfolgen und soll im Hygieneplan detailliert beschrieben werden.

Alle zugänglichen Endoskopkanäle und ihr Zubehör sollen neben dem Durchspülen auch einer manuellen Bürstenreinigung unterzogen werden.

Starker Konsens

\section{Empfehlung:}

Zur manuellen Reinigung von Endoskopen sollen Prozesschemikalien mit oberflächenaktiven, nicht schäumenden Substanzen wie Tenside, enzymatische Reiniger oder kombiniert reinigende-desinfizierende Lösungen verwendet werden.

Starker Konsens

\section{Empfehlung:}

Endoskope und ihr Zubehör sollen einer standardisierten und validierten Aufbereitung im RGD-E zugeführt werden.

Die manuelle Aufbereitung soll nur noch in Ausnahmefällen (z. B. Ausfall von RDG-E) durchgeführt werden.

Desinfektionsmittel sollen mindestens eine bakterizide, levurozide (wirksam gegen Hefen), tuberkulozide (wirksam gegen Mycobacterium terrae) und viruzide (wirksam gegen behüllte und unbehüllte Viren) Wirksamkeit umfassen.

Starker Konsens

\section{Empfehlung:}

Die Lagerung von Endoskopen soll vorzugsweise hängend, trocken, in einem geschlossenen Endoskopschrank erfolgen. Werden Trockenschränke verwendet, sollen sie regelmäßig gewartet und überprüft werden.

Starker Konsens

\section{Empfehlung:}

Die korrekte Durchführung der Aufbereitung soll Patientenbezogen dokumentiert werden. Dabei sollen die erfassten Prozessparameter und Freigabeentscheidung (freigebende Person und Charge) dokumentiert werden.

Starker Konsens

\subsubsection{Aufbereitung von endoskopischem Zusatzinstrumentarium Empfehlung:}

Medizinprodukte, die als Einmalmaterial gekennzeichnet sind, sollen nicht wieder aufbereitet werden.

Wiederaufbereitbare Zusatzinstrumente sollen einer standardisierten, validierten, Aufbereitung im Reinigungs- und Desinfektionsgerät (RDG) und abschließender Sterilisation unterzogen werden.

\section{Starker Konsens}

\subsection{Validierung und Routineüberprüfungen Empfehlung:}

Der Betreiber soll ein Qualitätsmanagement für die Hygiene zu etablieren, das die Validierung von RDG-E, die erneute Qualifikation und periodische Routineüberprüfungen beinhaltet.

Im Fall von technischen Problemen und bei Verdacht auf mögliche endoskopieassoziierte Infektionen soll der Betreiber das entsprechende Medizinprodukt (z. B. Endoskop, RGD-E) aus dem Betrieb nehmen, bis korrektive Maßnahmen ergriffen wurden und erneute mikrobiologische Untersuchungen, ggf. eine erneute Qualifikation zufriedenstellende Ergebnisse geliefert haben.

Starker Konsens

\subsection{Ergebnisqualität der Hygiene in der Endoskopie Statement:}

Die Qualität der Hygiene in der Endoskopie wird beeinflusst von der räumlich-apparativen Ausstattung und den personellen Voraussetzungen (Strukturqualität) sowie vom verantwortungsbewussten Verhalten des Personals und der Aufbereitungssicherheit (Prozessqualität) ( $\bullet$ Tab. 19)

Konsens

\begin{tabular}{|c|c|}
\hline Maßnahmen & $\begin{array}{l}\text { messbare Qualitätskriterien Hygiene in der } \\
\text { Endoskopie }\end{array}$ \\
\hline Personalschutz & $\begin{array}{l}\text { Verfügbarkeit und angemessener Gebrauch von } \\
\text { persönlicher Schutzausrüstung für Personal: } \\
\text { - Schutzhandschuhe } \\
\text { - Bereichskleidung } \\
\text { - Schutzkittel (langärmlig, hinter schließbar, } \\
\text { feuchtigkeitsabweisend), ggf. Plastikschürzen } \\
\text { - Gesichtsschutz (Schutzbrillen, Mundschutz, } \\
\text { Visier) } \\
\text { - Gesundheits- und Sicherheitsvorschriften } \\
\text { (z. B. Nadelstichverletzungen, Chemieunfälle) } \\
\text { - Hygieneplan, Standardarbeitsanweisungen } \\
\text { - regelmäßige betriebsärztliche } \\
\text { Gesundheitsüberwachung } \\
\text { - adäquater Impfschutz (HBV) } \\
\text { - Meldewesen inklusive Dokumentation und } \\
\text { Auswertung von Komplikationen, Infektionen, } \\
\text { Zwischenfällen, Unfällen, giftigen oder } \\
\text { allergischen Reaktionen }\end{array}$ \\
\hline Patientenschutz & $\begin{array}{l}\text { Verfügbarkeit und angemessener Gebrauch von } \\
\text { persönlicher Schutzmaßnahmen für Patienten, } \\
\text { z. B. Patientenschutzhemd, Patientenschutzho- } \\
\text { sen zur Koloskopie } \\
\text { Meldewesen inklusive Dokumentation und Aus- } \\
\text { wertung von Komplikationen, Infektionen, Zwi- } \\
\text { schenfällen, Unfällen, giftigen oder allergischen } \\
\text { Reaktionen } \\
\text { Anzahl der endoskopieassoziierten Infektionen }\end{array}$ \\
\hline $\begin{array}{l}\text { Aufbereitung von } \\
\text { Endoskopen und } \\
\text { Zusatzinstrumen- } \\
\text { tarium }\end{array}$ & $\begin{array}{l}\text { standardisierte und validierte Aufbereitung von } \\
\text { Endoskopen und anderen Medizinprodukten } \\
\text { (SOP) } \\
\text { validierte Aufbereitung im RGD-E und RDG } \\
\text { effektive Dokumentation und individuelle } \\
\text { Freigabe } \\
\text { Meldewesen inklusive Dokumentation und } \\
\text { Auswertung von Zwischenfällen, Unfällen, } \\
\text { Geräteausfällen, Defekten, etc. }\end{array}$ \\
\hline $\begin{array}{l}\text { Wartung, Validie- } \\
\text { rung und Routine- } \\
\text { überprüfungen }\end{array}$ & $\begin{array}{l}\text { Risikobewertung der Medizinprodukte } \\
\text { Wartung der RDG-E und Endoskope } \\
\text { Validierung und erneute Qualifikation von RDG-E, } \\
\text { RDG, Sterilisatoren und ggf. Trockenschränken } \\
\text { SOP und Dokumentation der Validierungen und } \\
\text { ggf. erneuter Qualifikation } \\
\text { SOP und Dokumentation der technischen und } \\
\text { mikrobiologischen Routinekontrollen }\end{array}$ \\
\hline
\end{tabular}


Kap. 7: Ergebnisqualität in der Endoskopie Allgemeine Qualitätsindikatoren (o Tab. 20)

$\nabla$

Tab. 20

\begin{tabular}{|c|}
\hline Allgemeine Qualitätsindikatoren Endoskopie \\
\hline präprozedural \\
\hline Dokumentation der Indikation für die Intervention (Kap. 5) \\
\hline $\begin{array}{l}\text { Durchführung eines standardisierten Risikoassessment vor dem } \\
\text { endoskopischen Eingriff (Kap. } 2.4 ; 3.4 \text { ) }\end{array}$ \\
\hline $\begin{array}{l}\text { Zeitgerechte Durchführung und Dokumentation von Patientenaufklä- } \\
\text { rung und Einverständnis (Kap. 3.1) }\end{array}$ \\
\hline Gabe einer Antibiotikaprophylaxe bei gegebener Indikation (Kap. 3.3) \\
\hline $\begin{array}{l}\text { Management von Gerinnungshemmender Medikation vor einem } \\
\text { endoskopischen Eingriff bei gegebener Indikation (Kap.3.2) }\end{array}$ \\
\hline Durchführung und Dokumentation eines Team-Time-Out (Kap. 3.4) \\
\hline intraprozedural \\
\hline $\begin{array}{l}\text { Durchführung einer Fotodokumentation von Landmarken und patholo- } \\
\text { gischen Befunden während des endoskopischen Eingriffs (Kap. 5) }\end{array}$ \\
\hline $\begin{array}{l}\text { Erfassung und Dokumentation der Dauer des endoskopischen Eingriffes } \\
\text { (Zeiterfassung, Kap. 5) }\end{array}$ \\
\hline $\begin{array}{l}\text { Durchführung und Dokumentation eines kontinuierlichen Vitalzeichen- } \\
\text { monitorings (Kap. 2.4) }\end{array}$ \\
\hline postprozedural \\
\hline $\begin{array}{l}\text { Durchführung eines standardisierten Entlassungsmanagements } \\
\text { (Kap. 3.4) }\end{array}$ \\
\hline Erstellung einer kompletten Befunddokumentation (Kap. 5) \\
\hline Erstellung und Dokumentation von Nachsorgeempfehlungen (Kap. 5) \\
\hline Dokumentation von Zubehör und Instrumentarien (Kap.5) \\
\hline Dokumentation von intra- und postprozeduralen Komplikationen (Kap. 5) \\
\hline $\begin{array}{l}\text { Erfassung der Frequenz von intra- und postprozeduralen Komplikationen } \\
\text { (interventionsspezifische Komplikationen, Kap. 4) }\end{array}$ \\
\hline
\end{tabular}

\section{Institute}

${ }^{1}$ Klinik und Poliklinik für Interdisziplinäre Endoskopie, Universitätsklinikum Hamburg-Eppendorf, Hamburg

2 DEGEA, Ulm

${ }^{3}$ Medizinische Klinik II, Gastroenterologie und Infektiologie, Klinikum Hanau, Hanau

${ }^{4}$ Asklepios Klinik Barmbek, Hamburg

${ }_{5}^{5}$ Dr. Heberer \& Kollegen, München

${ }^{6}$ DCCV, Berlin

7 Med. Klinik C, Klinikum der Stadt Ludwigshafen, Ludwigshafen

${ }^{8}$ Krankenhaus Märkisch Oderland GmbH, Klinik für Innere Medizin, Strausberg

${ }^{9}$ Klinik für Innere Medizin, Bethesda Krankenhaus Bergedorf, Hamburg

10 II. Medizinische Klinik und Poliklinik, Onkologisches Zentrum, Hubertus Wald Tumorzentrum, Universitätsklinikum Hamburg-Eppendorf, Hamburg

11 Klinik für Innere Medizin A, Universitätsmedizin Greifswald

12 DGVS, Berlin

13 Medizinische Klinik II/IV, Sana Klinikum, Offenbach

${ }^{14}$ Klinik für Allgemein- und Viszeralchirurgie, Universitätsklinikum, Münster

15 Gastroenterologische Facharztpraxis, Kassel

16 Medizinischen Klinik, Israelitisches Krankenhaus, Hamburg

17 FB Gastroenterologie, DKD Helios-Klinik, Wiesbaden

18 Medizinischen Klinik II. Klinikum am Gesundbrunnen, Heilbronn 


\section{Anhang: Checkliste Team-Time-Out für Endoskopische Prozeduren (o Tab. 21)}

$\nabla$

Tab. 21

\begin{tabular}{|c|c|c|c|c|c|}
\hline \multicolumn{6}{|l|}{$\begin{array}{l}\text { Patient: } \\
\text { Name/Geburtstag, Patienten ID etc. }\end{array}$} \\
\hline $\begin{array}{l}\text { Sign in: } \\
\text { - Übergabe an Endoskopie }\end{array}$ & $\checkmark$ & $\begin{array}{l}\text { Team-Time-Out } \\
\text { - direkt vor Beginn des Eingriff }\end{array}$ & $\checkmark$ & $\begin{array}{l}\text { Sign out } \\
\text { vor Verlegung/Entlassung }\end{array}$ & $\checkmark$ \\
\hline \multicolumn{2}{|c|}{ Die folgenden Kriterien werden geprüft/bestätigt } & \multicolumn{2}{|c|}{$\begin{array}{l}\text { Die folgenden Kriterien werden geprüft/ } \\
\text { bestätigt }\end{array}$} & \multicolumn{2}{|c|}{$\begin{array}{l}\text { Die folgenden Kriterien werden geprüft/ } \\
\text { bestätigt }\end{array}$} \\
\hline $\begin{array}{l}\text { Identifikation des Patienten } \\
\text { (Name, Geburtsdatum, Pat-ID) }\end{array}$ & $\square$ & $\begin{array}{l}\text { Teamvorstellung mit Name } \\
\text { und Aufgabe }\end{array}$ & $\square$ & $\begin{array}{l}\text { vollständige Dokumentation } \\
\text { inklusive Hinweise für Nachsorge }\end{array}$ & $\square$ \\
\hline Einverständniserklärung & $\square$ & $\begin{array}{l}\text { Identifikation des Patienten } \\
\text { (Name, Geburtsdatum, Pat-ID) }\end{array}$ & $\square$ & Präparate versorgt & $\square$ \\
\hline $\begin{array}{l}\text { alle Dokumente liegen vor (Labor, } \\
\text { Befunde, etc.) }\end{array}$ & $\square$ & $\begin{array}{l}\text { geplanter Eingriff (Indikation, } \\
\text { Fragestellung, etc.) }\end{array}$ & $\square$ & $\begin{array}{l}\text { Patientenzustand dokumentiert } \\
\text { (je nach Ausgangszustand) }\end{array}$ & a \\
\hline \multicolumn{6}{|l|}{ Risikobewertung: } \\
\hline $\begin{array}{l}\text { ASA-Klassifikation/Komorbidität } \\
\text { geprüft }\end{array}$ & $\square$ & $\begin{array}{l}\text { notwendige Instrumente } \\
\text { vorhanden? }\end{array}$ & $\square$ & & \\
\hline $\begin{array}{l}\text { Beatmungsprobleme, kardiorespirato- } \\
\text { rische Probleme }\end{array}$ & $\begin{array}{l}\square \text { ja } \\
\square \text { nein }\end{array}$ & \multirow{2}{*}{$\begin{array}{l}\text { Monitoring, Medikamente, } \\
\text { Equipment zum Atemwegsma- } \\
\text { nagement zur Verfügung und } \\
\text { überprüft }\end{array}$} & \multirow[t]{2}{*}{$\square$} & \multirow[t]{2}{*}{ Gab es Probleme beim Eingriff? } & \multirow[t]{2}{*}{$\begin{array}{l}\square \text { ja } \\
\text { घnein }\end{array}$} \\
\hline Allergien & $\begin{array}{l}\square \text { ja } \\
\square \text { nein }\end{array}$ & & & & \\
\hline Infektionen & $\begin{array}{l}\square \text { ja } \\
\square \text { nein }\end{array}$ & \multirow{2}{*}{$\begin{array}{l}\text { Endoskopiker nennt Besonder- } \\
\text { heiten zum Eingriff? } \\
\text { geplante Schritte } \\
\text { schwierige Schritte }\end{array}$} & \multirow[t]{2}{*}{$\begin{array}{l}\square \text { ja } \\
\text { anein }\end{array}$} & \multirow[t]{2}{*}{$\begin{array}{l}\text { Spez. Hinweise zur Nachsorge } \\
\text { gegeben? }\end{array}$} & \multirow[t]{2}{*}{$\begin{array}{l}\text { वja } \\
\text { घnein }\end{array}$} \\
\hline Antikoagulantien & $\begin{array}{l}\square \text { ja } \\
\square \text { nein }\end{array}$ & & & & \\
\hline Glaukom & $\begin{array}{l}\square \text { ja } \\
\square \text { nein }\end{array}$ & \multirow[t]{2}{*}{$\begin{array}{l}\text { Besonderheiten zur Sedierung } \\
\text { und Lagerung? }\end{array}$} & $\begin{array}{l}\square \text { ja } \\
\square \text { nein }\end{array}$ & \multirow[t]{2}{*}{$\begin{array}{l}\text { Spez. Hinweise zur Verlegung/ } \\
\text { Entlassung? }\end{array}$} & \multirow[t]{2}{*}{$\begin{array}{l}\square \text { ja } \\
\square \text { nein }\end{array}$} \\
\hline Nüchtern/Vorbereitung & $\begin{array}{l}\square \text { ja } \\
\square \text { nein }\end{array}$ & & & & \\
\hline Unterschrift & & Unterschrift & & Unterschrift & \\
\hline
\end{tabular}

